\title{
Classification of three-family grand unification in string theory. II. The SU(5) and SU(6) models
}

\author{
Zurab Kakushadze* \\ Lyman Laboratory of Physics, Harvard University, Cambridge, Massachusetts 02138 \\ S.-H. Henry Tye ${ }^{\dagger}$ \\ Department of Physics, Northeastern University, Boston, Massachusetts 02115 \\ and Newman Laboratory of Nuclear Studies, Cornell University, Ithaca, New York 14853-5001
}

(Received 14 January 1997)

\begin{abstract}
Requiring that supersymmetric SU(5) and SU(6) grand unifications in the heterotic string theory must have three chiral families, adjoint (or higher representation) Higgs fields in the grand unified gauge group, and a non-Abelian hidden sector, we construct such string models within the framework of free conformal field theory and asymmetric orbifolds. Within this framework, we construct all such string models via $\mathbf{Z}_{6}$ asymmetric orbifolds that include a $\mathbf{Z}_{3}$ outerautomorphism, the latter yielding a level-three current algebra for the grand unification gauge group $\mathrm{SU}(5)$ or $\mathrm{SU}(6)$. We then classify all such $\mathbf{Z}_{6}$ asymmetric orbifolds that result in models with a non-Abelian hidden sector. All models classified in this paper have only one adjoint (but no other higher representation) Higgs field in the grand unified gauge group. This Higgs field is neutral under all other gauge symmetries. The list of hidden sectors for three-family SU(6) string models are $\mathrm{SU}(2), \mathrm{SU}(3)$, and $\mathrm{SU}(2) \otimes S U(2)$. In addition to these, three-family SU(5) string models can also have an SU(4) hidden sector. Some of the models have an apparent anomalous U(1) gauge symmetry. [S0556-2821(97)01212-5]

PACS number(s): 11.25.Mj, 12.10.Dm, 12.60.Jv
\end{abstract}

\section{INTRODUCTION}

For superstring theory to describe nature, it must contain the standard model of strong and electroweak interactions as part of its low energy (i.e., below the string scale) effective field theory. Among the various possibilities, grand unified theory (GUT) is particularly attractive because it is a truly unified theory with one gauge coupling in the low energy effective theory. Various GUT's in string theory have been extensively studied [1]. Since nature seems to have only three chiral families of quarks and leptons, it is reasonable to impose this condition in model building. In earlier papers [2-5], we have considered explicit realization of such threefamily GUT's in the heterotic string theory. The number of such possibilities turns out to be quite limited. In [5], we present a classification of three-family $\mathrm{SO}(10)$ and $\mathrm{E}_{6}$ grand unified heterotic string models, within the framework of perturbative string theory. In this paper, we present a classification of three-family SU(5) and SU(6) grand unified heterotic string models, within a somewhat more restricted framework. Together with [5], this paper completes our classification of all three-family grand unification within the framework of asymmetric orbifolds in conformal (free) field theory [6], as described below.

For the grand unified gauge symmetry to be broken spontaneously to the standard model in the low energy effective field theory, it is well known that an adjoint (or other appropriate representation) Higgs field is needed. So the requirement of such a massless Higgs field will be imposed in string

\footnotetext{
*Electornic address: zurab@string.harvard.edu

†Electronic address: type@hepth.cornell.edu
}

model building. It is most natural to have space-time supersymmetry in string models, which will also be imposed. This supersymmetry is expected to be broken via dynamical supersymmetry breaking in the hidden sector, which requires an asymptotically free hidden sector. This is then transmitted to the observable sector via gravity or other messenger and/or intermediate sector [7]. So we are lead to impose the following constraints on grand unified string model building: (i) $N=1$ space-time supersymmetry; (ii) three chiral families of fermions in the GUT gauge group; (iii) adjoint (and/or other appropriate) Higgs fields in GUT; (iv) non-Abelian hidden sector.

Imposing these constraints on string model building within the framework of asymmetric orbifolds, we argue in [5] that, in the classification of three-family $\mathrm{SO}(10)$ and $\mathrm{E}_{6}$ string models, that we need only consider $\mathbf{Z}_{6}$ asymmetric orbifolds that include a $\mathbf{Z}_{3}$ outer-automorphism. So the classification problem is reduced to the classification of such $\mathbf{Z}_{6}$ asymmetric orbifold models that have the above properties. This is carried out in [5] and in this paper. It is important to point out a key difference between the construction of the $\mathrm{SO}(10)$ and $\mathrm{E}_{6}$ versus the $\mathrm{SU}(5)$ string models. As explained in [5], these GUT gauge symmetries are to be realized at level-three current (Kac-Moody) algebras. Most GUT realizations of these higher-level gauge symmetries $G_{k}$ employ the so-called diagonal embeddings $G_{k} \subset G$ $\otimes G \otimes \cdots \otimes G=G^{k}$ (here, the superscript $k$ indicates the power, while the subscript $k$ indicates the level). A $\mathbf{Z}_{k}$ outerautomorphism of $G^{k}$ yields $G_{k}$ (plus an appropriate coset). It was pointed out by Dienes and March-Russell [8] that, for $G_{k}=\mathrm{SO}(10)_{3},\left(\mathrm{E}_{6}\right)_{3}$, or $\mathrm{SU}(6)_{3}$, this diagonal embedding is the maximal embedding. So a classification of $\mathbf{Z}_{6}$ asymmetric orbifolds that yield three-family $\mathrm{SO}(10)$, 
$\mathrm{SU}(6)$, and $\mathrm{E}_{6}$ string models may be considered as a classification of three-family $\mathrm{SO}(10), \mathrm{SU}(6)$, and $\mathrm{E}_{6}$ string models. However, for $\mathrm{SU}(5)_{3}$, there is a more economical embedding, namely, $\mathrm{SU}(5)_{3} \subset \mathrm{SU}(10)_{1}$. This nondiagonal embedding opens the possibility that the $\mathbf{Z}_{3}$ outerautomorphism, and hence the $\mathbf{Z}_{6}$ asymmetric orbifolds, may not exhaust all possible three-family SU(5) string models. In this sense, the list of three-family SU(5) string models given in this work may be incomplete; that is, the classification of three-family SU(5) string models given in this paper includes only those that are reachable via the diagonal embedding.

In [5], we gave a list of three-family $\mathrm{SO}(10)$ and $\mathrm{E}_{6}$ string models. Since the adjoint Higgs field is a modulus in the string moduli space, some three-family $\mathrm{SU}(5)$ and $\mathrm{SU}(6)$ string models can be easily obtained by giving the adjoint Higgs field in the $\mathrm{E}_{6}$ model an appropriate vacuum expectation value (VEV). In a similar fashion, three-family $\mathrm{SU}(5)$ string models can be easily obtained from the $\mathrm{SO}(10)$ models. Although these models are interesting on their own, they are rather trivial from the model-building point of view. In this work, we shall focus mainly on the three-family SU(5) and SU(6) string models that cannot be reached in this way. In particular, we are interested in models with an enhanced hidden sector. Since the construction of SU(6) string models is closely related to that of the SU(5) models, their classification should go together.

Besides the properties that resulted from the constraints imposed in string model building, all the three-family GUT string models share the following properties: there is only one adjoint (plus some lower, but no higher, representation) Higgs field; there is an intermediate or horizontal gauge symmetry.

In addition, there are three key phenomenologically interesting features that distinguish the three-family $\mathrm{SU}(5)$ and $\mathrm{SU}(6)$ string models from the three-family $\mathrm{SO}(10)$ and $\mathrm{E}_{6}$ string models.

(i) Recall that the three-family $\mathrm{SO}(10)$ and $\mathrm{E}_{6}$ string models have either four left-handed and one right-handed families, or five left-handed and two right-handed families. In contrast, some of the three-family SU(5) and SU(6) string models have truly three chiral families. For example, one model has 3 left-handed 10's, 12 left-handed and 9 righthanded $\overline{\mathbf{5}}$ 's of SU(5), yielding 3 chiral families and 9 Higgs multiplets.

(ii) In contrast to the three-family $\mathrm{SO}(10)$ and $\mathrm{E}_{6}$ string models, some of the three-family $\mathrm{SU}(5)$ and $\mathrm{SU}(6)$ string models have an apparent anomalous $\mathrm{U}(1)$ gauge symmetry; that is, the anomaly contributions of the U(1) charged massless fermions do not add up to zero. Here, the anomaly cancellation is accomplished by the presence of some massless scalar fields in the model. This is the so-called GreenSchwarz mechanism in string theory, and this anomalous $\mathrm{U}(1)$ gauge symmetry is expected to be broken by the FayetIliopoulos term [9]. The U(1) breaking happens at some scale slightly below the string scale, providing a natural scale rather close to the grand unification scale naively expected. Presumably, a number of scalar fields will develop vacuum expectation values without breaking supersymmetry.

(iii) A simple robust way to stabilize the dilaton expectation value is to have a semi-simple hidden sector, with more than one gaugino condensate [10]. So it is interesting to ask if one can get a hidden sector with more than one gaugino condensate in the three-family GUT string model construction. In [5], we saw that the biggest (and only) asymptotically free (at the string scale) hidden sector gauge symmetry was SU(2). On the other hand, there are three-family SU(5) and $\mathrm{SU}(6)$ string models that allow a hidden sector with an asymptotically free $\mathrm{SU}(2) \otimes S U(2)$ semisimple gauge group at the string scale. In fact, these two SU(2)'s have different matter contents, so the runnings of their couplings will be somewhat different. This is believed to be a desirable feature. Naively, because of the large matter contents, these SU(2)'s are expected to get strong at very low energy scales. However, this is not expected to happen. Instead, the anomalous $\mathrm{U}(1)$ gauge symmetry breaking is expected to give masses to some of the SU(2) multiplets. Furthermore, these $\mathrm{SU}(2)$ couplings $\alpha_{2}$ are three times that of the grand unified gauge coupling. So, the gauge couplings of these two $\mathrm{SU}(2)$ 's will become large at rather high scales. However, they will become strong at different scales, each with its own gaugino condensate [7]. A careful analysis of specific models is clearly needed to see if supersymmetry breaking happens in any of the models in a way that will stabilize the dilaton expectation value to a reasonable value [10].

The list of gauge symmetries of the three-family SU(6) string models is $\mathrm{SU}(2)_{1} \otimes \mathrm{SU}(2)_{1} \otimes \mathrm{SU}(6)_{3} \otimes \mathrm{U}(1)^{3}$, $\mathrm{SU}(3)_{1} \otimes \mathrm{SU}(6)_{3} \otimes \mathrm{U}(1)^{3}, \quad$ and $\quad \mathrm{SU}(2)_{1} \otimes \mathrm{SU}(6)_{3} \otimes \mathrm{U}(1)^{4}$. The list of gauge symmetries of the three-family SU(5) string models includes the above list where the $\mathrm{SU}(6)_{3}$ is replaced by $\mathrm{SU}(5)_{3} \otimes \mathrm{U}(1)$. In addition, there is an $\mathrm{SU}(5)$ string model with gauge symmetry, $\mathrm{SU}(4)_{1}$ $\otimes \mathrm{SU}(2)_{1} \otimes \mathrm{SU}(5)_{3} \otimes \mathrm{U}(1)^{4}$, where the $\mathrm{SU}(4)$ plays the role of a hidden sector. The lists of three-family GUT string models are given in this paper and in [5]. Some of these models have been presented earlier [2-4]. The massless spectra of all the three-family $\mathrm{E}_{6}$ and $\mathrm{SO}(10)$ string models are given in tables in [5]. The massless spectra of some of the threefamily SU(5) and SU(6) string models are given in tables in this paper. Given the orbifolds, the massless spectra of the remaining models can be easily written down, following the rules described below.

The rest of this paper is organized as follows. In Sec. II we discuss the general grounds underlying the classification of three-family SU(5) and SU(6) string models. Next, we turn to the construction of the models. Since three-family $\mathrm{E}_{6}$ and $\mathrm{SO}(10)$ models have been constructed and classified in [5] already, it is easiest if we simply convert the $\mathrm{E}_{6}$ and $\mathrm{SO}(10)$ models to the SU(5) and SU(6) models with appropriate Wilson lines. This approach is discussed in Sec. III. The classification is given in Sec. IV, with the massless spectra of the more interesting string models given in the tables. In Sec. V, we briefly discuss the moduli space of the models and the ways they are connected to each other. We conclude with some remarks in Sec. VI.

\section{PRELIMINARIES}

The general grounds on which the classification is based have been explained in [5]. First, let us briefly review them. Then we shall discuss the issues particular to the SU(5) case.

(i) Phenomenologically, the gauge coupling at the grand unification scale is weak. Since this scale is quite close to the 
string scale, the coupling at the string scale is also expected to be weak. This means conformal field theory is valid. So string model building can be restricted to the fourdimensional heterotic string theory using conformal field theories and current (or Kac-Moody) algebras. Our framework throughout will be the asymmetric orbifold construction $[3,6]$.

(ii) By the three chiral families constraint, we mean that the net number of chiral families (defined as the number of left-handed families minus the number of the right-handed families) must be three.

(iii) As we mentioned earlier, the hidden sector is required so that dynamical supersymmetry breaking may occur. This means the hidden sector must contain a non-Abelian gauge symmetry.

(iv) The requirement of adjoint Higgs in $N=1$ supersymmetric GUT implies the grand unified gauge symmetry must be realized via a higher-level current algebra.

An exhaustive search in the level-two algebras [1] indicates that the three chiral families plus the adjoint Higgs constraints require level-three or higher current algebras for the grand unified gauge symmetry. So it is natural to go to level-three models. Since their construction typically requires a $\mathbf{Z}_{3}$ outerautomorphism, this can be part of a $\mathbf{Z}_{3}$ orbifold, which has an odd number of fixed points. So there is a chance to construct models with three chiral families via $\mathbf{Z}_{3}$ orbifolds. Naively, one might expect that three-family string GUT's can be constructed with a single $\mathbf{Z}_{3}$ twist, since the latter can, at least in principle, be arranged to have three fixed points. This, however, turns out not to be the case [5]. So, to obtain a model with three chiral families, we are lead to consider asymmetric $\mathbf{Z}_{6}$ orbifolds.

As mentioned in Sec. I, the common way to realize a higher-level gauge symmetry $G_{k}$ employs the so-called diagonal embeddings, that is, a $\mathbf{Z}_{k}$ outerautomorphism of $G^{k}$ yields $G_{k}$ (plus an appropriate coset). It was pointed out by Dienes and March-Russell [8] that, for $G_{k}=\operatorname{SO}(10)_{3}$, $\left(\mathrm{E}_{6}\right)_{3}$, or $\mathrm{SU}(6)_{3}$, this diagonal embedding (i.e., $G_{k} \subset G^{k}$ ) is the maximal embedding. So a classification of $\mathbf{Z}_{6}$ asymmetric orbifolds that yield three-family $\mathrm{SO}(10), \mathrm{SU}(6)$, and $\mathrm{E}_{6}$ string models may be considered as a classification of threefamily $\mathrm{SO}(10), \mathrm{SU}(6)$, and $\mathrm{E}_{6}$ string models. However, this is not the case for $\mathrm{SU}(5)_{3}$. Besides the diagonal embedding, there is another embedding, namely, $\mathrm{SU}(5)_{3} \subset \mathrm{SU}(10)_{1}$. Since the central charge of $\mathrm{SU}(10)_{1}$ is 9 , while that of $\mathrm{SU}(5)^{3}$ is 12 , the embedding in $\mathrm{SU}(10)_{1}$ is clearly more economical. Since the constraint of three chiral families requires a $\mathbf{Z}_{6}$ twist, we can ask if there is a $\mathbf{Z}_{6}$ twist of $\mathrm{SU}(10)_{1}$ that yields $\mathrm{SU}(5)_{3}$. Without even worrying about string consistency, it is a straightforward, but somewhat tedious, exercise to show that no $\mathbf{Z}_{3}$ or $\mathbf{Z}_{6}$ twisting of the $\mathrm{SU}(10)_{1}$ can yield $\mathrm{SU}(5)_{3}$. This means that this nondiagonal embedding opens the possibility that the $\mathbf{Z}_{3}$ outerautomorphism, and hence the $\mathbf{Z}_{6}$ asymmetric orbifolds, may not exhaust all possible three-family $\mathrm{SU}(5)$ string models. In this sense, the list of three-family SU(5) string models given in this work may be incomplete. That is, the classification of three-family SU(5) string models given in this paper includes only those that are reachable via the diagonal embedding. It is a very interesting and challenging problem to see if threefamily SU(5) string models can be constructed via such a nondiagonal embedding. Since $\mathrm{SU}(5)$ is a smaller gauge group than the other grand unified groups, one may also want to ask the same question about higher-level SU(5)'s.

The above discussion reduces this restricted classification problem to the classification of $\mathbf{Z}_{6}$ asymmetric orbifold models with the following phenomenological requirements (that translate into stringent constraints in the actual string model building) to be imposed: $N=1$ space-time supersymmetry, level-3 GUT via diagonal embedding, three chiral families in GUT, non-Abelian hidden sector. It is this classification problem that we solve in [5] and this paper. All of the threefamily $\mathrm{SU}(5)$ and $\mathrm{SU}(6)$ string models [that cannot be obtained by simple spontaneous symmetry breaking of $\mathrm{SO}(10)$ and $\mathrm{E}_{6}$ string models] are given in this paper, and their massless spectra are presented in the tables. Now the classification project consists of three steps: (i) list all suitable $N=4$ Narain models [11], (ii) list all $\mathbf{Z}_{6}$ asymmetric twists that can act on each Narain model, (iii) work out the massless spectrum in each orbifold to check its properties. As in [5], the classification only distinguishes different string models by their tree-level massless spectra. It is well known that some orbifolds, though they look different at first sight, nevertheless yield identical models. Even when their massless spectra are identical, some particular massless particles may appear in different sectors in different orbifolds. These models are simply related by $T$ duality. It happens that their interactions are easiest to determine if we consider both orbifolds simultaneously.

The gauge coupling of a given group $G$ in the model at a scale $\mu$ below the string scale $M_{s}$ is related to it via

$$
1 / \alpha_{G}(\mu)=k_{G} / \alpha_{\text {string }}+\left(b_{0} / 4 \pi\right) \ln \left(M_{s}^{2} / \mu^{2}\right),
$$

where $k_{G}$ is the level of the gauge group. For a $\mathrm{U}(1)$ gauge theory, $1 / k=2 r^{2}$ if the $\mathrm{U}(1)$ charge is normalized so that the lowest allowed value is \pm 1 (with conformal highest weight $r^{2} / 2$ ), and $r$ is the compactification radius of the corresponding chiral world-sheet boson. The constant $b_{0}$ is the one-loop coefficient of the $\beta$ function.

A typical model will have some moduli that can be varied (within some ranges) without affecting its massless spectrum. Here we shall not distinguish such models. We will, however, discuss the moduli space in which these models sit.

\section{MODEL CONSTRUCTION}

Starting from a consistent heterotic string model, typically a Narain model, a new model can be generated by performing a consistent set of twists and shifts on it. First, these twists and shifts must be consistent with the symmetry of the momentum lattice. To obtain a new consistent string model, the following conditions are imposed on its one-loop partition function in the light-cone gauge: (i) one-loop modular invariance, (ii) world-sheet supersymmetry, and (iii) the physically sensible projection. This last condition means the contribution of a space-time fermionic (bosonic) degree of freedom to the partition function counts as minus (plus) one. In all cases that can be checked, this condition plus the oneloop modular invariance and factorization imply multi-loop modular invariance.

A set of twists and shifts can be organized into a set of 
vectors. The rules of consistent asymmetric orbifold model construction can then be written as constraints on these vectors, as done in [3]. All sets of vectors given in this and the next sections, i.e., the Wilson lines $\left(U_{1}, U_{2}\right), A_{i}$ and $B_{i}$, and the $\mathbf{Z}_{6}$ orbifolds $\left(T_{3}, T_{2}\right)$, have been checked to satisfy these consistency constraints, provided appropriate choices of the structure constants are picked. The structure constants dictate which sets of states are projected out. The spectrum of the resulting model can be obtained from the spectrum generating formula. It is straightforward, but somewhat tedious, to work out the massless spectrum in each model. Since threefamily $\mathrm{E}_{6}$ and $\mathrm{SO}(10)$ models have been constructed and classified in [5] already, the construction of three-family $\mathrm{SU}(5)$ and SU(6) models is easiest if we simply convert the $\mathrm{E}_{6}$ and $\mathrm{SO}(10)$ models to them with appropriate Wilson lines. We shall borrow heavily from [5], and follow its notations. Models labeled with $N, E, T, S$, and $F$ are Narain, $\mathrm{E}_{6}, \mathrm{SO}(10), \mathrm{SU}(6)$, and $\mathrm{SU}(5)$ string models, respectively.

There are two procedures to convert the $\mathrm{E}_{6}$ and $\mathrm{SO}(10)$ models to $\mathrm{SU}(5)$ and $\mathrm{SU}(6)$ models.

(i) Take one of the $\mathrm{E}_{6}$ or $\mathrm{SO}(10)$ models given in [5], which is obtained via a particular $\mathbf{Z}_{6}$ orbifold on a specific Narain model. Introduce an appropriate Wilson line (compatible with the Narain model and the $\mathbf{Z}_{6}$ orbifold) to act on this Narain model to obtain a new Narain model. Such a Wilson line turns out to have order 3 . Then, performing the same $\mathbf{Z}_{6}$ orbifold on this new Narain model, an SU(5) or SU(6) model results.

(ii) Start from the the same original Narain model chosen above. Instead of performing a $\mathbf{Z}_{6}$ orbifold on it, one may combine the (order 3 ) Wilson line with the $\mathbf{Z}_{6}$ orbifold to form a $\mathbf{Z}_{6} \otimes \mathbf{Z}_{3}$ orbifold. Depending on the choice of the new structure constants (which determine the relative phases between the various sectors), the action of this orbifold on the Narain model can yield the same $\mathrm{SU}(5)$ or SU(6) model as above. It turns out that, sometimes, there exists an alternative choice of the structure constants, yielding a different $\mathrm{SU}(5)$ or SU(6) model.

Finally, we work out the massless spectrum of the final model to check if it satisfies the phenomenological constraints. By relating each $\mathrm{SU}(5)$ or $\mathrm{SU}(6)$ model to an $\mathrm{E}_{6}$ or $\mathrm{SO}(10)$ model, the tedious working out of the massless spectrum is substantially simplified. Since the consistent $\mathbf{Z}_{6}$ orbifolds have been classified in [5], the classification of SU(5) and $\mathrm{SU}(6)$ string models is reduced to a classification of the additional Wilson lines to be added.

Let us illustrate these procedures by briefly describing the construction of the three-family SU(5) models, namely the $F 1$ and the $F 2$ models. The first procedure involves the following steps: (i) Starting from the $N=4$ supersymmetric Narain model, with $\mathrm{SU}(3) \otimes \mathrm{SO}(8) \otimes \mathrm{SO}(32)$ gauge symmetry, we introduce Wilson lines to convert it to another Narain model with gauge symmetry $\mathrm{SU}(3) \otimes \mathrm{SO}(8) \otimes \mathrm{SO}(10)^{3} \otimes \mathrm{SO}(2), \quad$ namely, the $N 1(1,0)$ model. (ii) An appropriate $\mathbf{Z}_{6}$ orbifold on this $N 1(1,0)$ model yields the three-family $\mathrm{SO}(10)$ model, with gauge symmetry $\mathrm{SU}(2)_{1} \otimes \mathrm{SU}(2)_{3} \otimes \mathrm{SO}(10)_{3} \otimes \mathrm{U}(1)^{3}$. This is the $T 1(1,0)$ model. (iii) An appropriate Wilson line acting on the $N 1(1,0)$ model yields another Narain model, namely the $N 6$ model. The same $\mathbf{Z}_{6}$ orbifold on this $N 6$ model yields the three-family $\mathrm{SU}(5)$ model, namely the $F 1$ model. The rest of this section gives a more detailed description of this construction and the alternative construction, which yields both the $F 1$ and the $F 2$ models.

Consider the Narain model with the momenta of the internal bosons spanning an even self-dual Lorentzian lattice $\Gamma^{6,22}=\Gamma^{6,6} \otimes \Gamma^{16}$. Here $\Gamma^{16}$ is the spin (32)/Z $\mathbf{Z}_{2}$ lattice. The $\Gamma^{6,6}$ is the momentum lattice corresponding to the compactification on a six-torus defined by $X_{I}=X_{I}+E_{I}$. The dot product of the vectors $E_{I}$ defines the constant background metric $G_{I J}=E_{I} \cdot E_{J}$. There is also the antisymmetric background field $B_{I J}$. The components of $G_{I J}$ and $B_{I J}$ parametrize the 36 -dimensional moduli space of the $\Gamma^{6,6}$ lattice. We are only interested in the subspace of the moduli space that has (1) appropriate $\mathbf{Z}_{3}$ symmetries, upon which we have to perform a $\mathbf{Z}_{3}$ twist later, and (2) an enhanced gauge symmetry so that, after the orbifold, the hidden sector can have maximal gauge symmetry. With these constraints, it will be suffice to consider a two-dimensional subspace of the general moduli space. This subspace is parametrized by the moduli $h$ and $f$, and the vectors $E_{I}$ (and also their duals $\widetilde{E}^{I}$ defined so that $E_{I} \cdot \widetilde{E}^{J}=\delta_{I}^{J}$ ) can be expressed in terms of the SU(3) root and weight vectors $e_{i}$ and $\widetilde{e^{i}}(i=1,2)$ :

$$
\begin{gathered}
E_{1}=\left(e_{1}, 0,0\right), \quad E_{2}=\left(e_{2}, 0,0\right), \\
E_{3}=\left(0, e_{1}, 0\right), \quad E_{4}=\left(0, e_{2}, 0\right), \\
E_{5}=\left(f \widetilde{e^{2}},-h \widetilde{e}^{2}, g e_{1}\right), \quad E_{6}=\left(-f \widetilde{e}^{-1}, h \widetilde{e}^{1}, g e_{2}\right),
\end{gathered}
$$

where $g=\sqrt{1-\left(f^{2}+h^{2}\right) / 3}$. The components of the antisymmetric tensor are chosen to be $2 B_{I J}=\frac{1}{2} G_{I J}$ for $I<J$ and $2 B_{I J}=-\frac{1}{2} G_{I J}$ for $I>J$. We shall call these Narain models as $N(h, f)$. With the above choice of the $\Gamma^{6,6}$ lattice, the Narain model $N(h, f)$ has the gauge symmetry $R(h, f) \otimes \mathrm{SO}(32)$. Let us consider the region $0 \leqslant h, f \leqslant 1$. A generic point in the moduli space of $N(h, f)$ has $R(h, f)=\mathrm{SU}(3) \otimes \mathrm{SU}(3) \otimes \mathrm{U}(1)^{2}$. There are four isolated points with enhanced gauge symmetry: $R(0,0)=$ $\mathrm{SU}(3) \otimes \mathrm{SU}(3) \otimes \mathrm{SU}(3), \quad R(1,0)=R(0,1)=\mathrm{SU}(3) \otimes \mathrm{SO}(8)$, and $R(1,1)=\mathrm{E}_{6}$.

To be specific, let us consider the $(h, f)=(1,0)$ case. Here we are writing the Wilson lines as shift vectors in the $\Gamma^{6,22}$ lattice. The shift vectors $U_{1}$ and $U_{2}$ to be introduced are order-2 shifts that break $\mathrm{SO}(32)$ to $\mathrm{SO}(10)^{3} \otimes \mathrm{SO}(2)$ :

$$
\begin{aligned}
& U_{1}=\left(e_{1} / 2, a_{1}, b_{1} \| 0,0,0\right)(\mathbf{s}|\mathbf{0}| \mathbf{0} \mid \bar{S}), \\
& U_{2}=\left(e_{2} / 2, a_{2}, b_{2}|| 0,0,0\right)(\mathbf{0}|\mathbf{s}| \mathbf{0} \mid \bar{S}) .
\end{aligned}
$$

The $U_{1}$ and $U_{2}$ are order-2 $\left(\mathbf{Z}_{2}\right)$ shifts. The first three entries correspond to the right-moving complex world-sheet bosons. The next three entries correspond to the left-moving complex world-sheet bosons. Together they form the six-torus. The remaining 16 left-moving world-sheet bosons generate the $\operatorname{spin}(32) / \mathbf{Z}_{2}$ lattice. The $\mathrm{SO}(32)$ shifts are given in the $\mathrm{SO}(10)^{3} \otimes \mathrm{SO}(2)$ basis. In this basis, $\mathbf{0}(0)$ stands for the null vector, $\mathbf{v}(V)$ is the vector weight, whereas $\mathbf{s}(S)$ and $\overline{\mathbf{s}}(\bar{S})$ are the spinor and antispinor weights of $\mathrm{SO}(10)[\mathrm{SO}(2)]$. [For 
$\mathrm{SO}(2), V=1, S=1 / 2$ and $\bar{S}=-1 / 2$.] Here the four component vectors $\left(a_{1}, b_{1}\right)$ and $\left(a_{1}, b_{1}\right)$ are the spinor $\mathbf{s}$ and conjugate c weights of $\mathrm{SO}(8)$, respectively. This model has $\mathrm{SU}(3) \otimes \mathrm{SO}(8) \otimes \mathrm{SO}(10)^{3} \otimes \mathrm{SO}(2)$ gauge symmetry. This is the $N 1(1,0)$ model.

Next we can perform a $\mathbf{Z}_{6}$ orbifold on the above $N 1(1,0)$ model. It is easier to follow the construction by decomposing the $\mathbf{Z}_{6}$ orbifold into a $\mathbf{Z}_{3}$ twist and a $\mathbf{Z}_{2}$ twist:

$$
\begin{gathered}
T_{3}=(\theta, \theta, \theta \| 0, \theta, \theta)(\mathcal{P} \mid 2 / 3), \\
T_{2}=\left(0, \sigma, \sigma \| e_{1} / 2, \sigma, \sigma\right)\left(0^{15} \mid 0\right) .
\end{gathered}
$$

Each $\theta$ in $T_{3}$ is a $\mathbf{Z}_{3}$ twist (that is, a $2 \pi / 3$ rotation) that acts only on a complex world-sheet boson. So the first $\theta$ acts on the right-moving part of the $\Gamma^{2,2}$ [i.e., the $\left.\mathrm{SU}(3)\right]$ lattice and the corresponding oscillator excitations, while the leftmoving part is untouched. This is an asymmetric orbifold. The $\Gamma^{4,4}$ [i.e., the $\mathrm{SO}(8)$ ] lattice is twisted symmetrically by the $\mathbf{Z}_{3} \otimes \mathbf{Z}_{3} \theta$ twist. The three $\mathrm{SO}(10)$ s are permuted by the action of the $\mathbf{Z}_{3}$ outerautomorphism twist $\mathcal{P}$ : $\phi_{1}^{I} \rightarrow \phi_{2}^{I} \rightarrow \phi_{3}^{I} \rightarrow \phi_{1}^{I}$, where the real bosons $\phi_{p}^{I}, I=1, \ldots, 5$, correspond to the $p$ th $\mathrm{SO}(10)$ subgroup, $p=1,2,3$. We can define new bosons $\varphi^{I} \equiv(1 / \sqrt{3})\left(\phi_{1}^{I}+\phi_{2}^{I}+\phi_{3}^{I}\right)$; the other ten real bosons are complexified via linear combinations $\Phi^{I} \equiv(1 / \sqrt{3})\left(\phi_{1}^{I}+\omega \phi_{2}^{I}+\omega^{2} \phi_{3}^{I}\right) \quad$ and $\quad\left(\Phi^{I}\right)^{\dagger} \equiv(1 / \sqrt{3})\left(\phi_{1}^{I}\right.$ $\left.+\omega^{2} \phi_{2}^{I}+\omega \phi_{3}^{I}\right)$, where $\omega=\exp (2 \pi i / 3)$. Under $\mathcal{P}, \varphi^{I}$ is invariant, while $\Phi^{I}\left[\left(\Phi^{I}\right)^{\dagger}\right]$ are eigenstates with eigenvalue $\omega^{2}(\omega)$. The $\mathbf{Z}_{3}$ invariant states form irreducible representations (irreps) of $\mathrm{SO}(10)_{3}$. Finally, string consistency requires the inclusion of the $2 / 3$ shift in the $\mathrm{SO}(2)$ lattice. This simply changes the radius of this world-sheet boson. The $\sigma$ in the $T_{2}$ twist is a $\pi$ rotation of the corresponding two chiral world-sheet bosons. Thus, $\sigma$ is a $\mathbf{Z}_{2}$ twist. The left-moving momenta of $\Gamma^{2,2}$ is shifted by $e_{1} / 2$ (i.e., half a root vector), while the $\Gamma^{16}$ is left untouched.

The resulting $N=1$ supersymmetric three-family $\mathrm{SO}(10)$ model, the $T 1(1,0)$ model, has $\mathrm{SU}(2)_{1} \otimes$ $\mathrm{SU}(2)_{3} \otimes \mathrm{SO}(10)_{3} \otimes \mathrm{U}(1)^{3}$ gauge symmetry (the subscripts indicate the levels of the corresponding Kac-Moody algebras). Its massless spectrum is given in [5]. This model is completely anomaly free, and its hidden sector is $\mathrm{SU}(2)_{1}$, whereas the observable sector is $\mathrm{S} 0(10)_{3} \otimes \mathrm{U}(1)^{2}$. The remaining $\mathrm{SU}(2)_{3} \otimes \mathrm{U}(1)$ plays the role of the messenger/intermediate sector, or horizontal symmetry. The net number of the chiral $\mathrm{SO}(10)_{3}$ families in this model is $5-2=3$. The details of the construction of this model can be found in [3].

Now, the final $F 1$ model can be obtained by introducing the following Wilson line to the $T 1(1,0)$ model:

$$
\left(0,0,0 \| \widetilde{e}^{2},-\widetilde{e}^{2}, 0\right)\left(\left(\frac{1}{3} \frac{1}{3} \frac{1}{3} \frac{1}{3} \frac{2}{3}\right)^{3} \mid 0\right) .
$$

This Wilson line is called $A_{6}$ in the next section. Here, the shift $1 / 31 / 31 / 31 / 32 / 3$ in the $\mathrm{SO}(10)$ lattice breaks $\mathrm{SO}(10)$ to $\mathrm{SU}(5)^{3} \otimes \mathrm{U}(1)$. Let us introduce this Wilson line to the $N 1(1,0)$ model to obtain a new Narain model, namely, the $N 6$ model. In the unshifted sector of this N6 model, the gauge group is broken from $\mathrm{SU}(3)$ $\otimes \mathrm{SO}(8) \otimes \mathrm{SO}(10)^{3} \otimes \mathrm{SO}(2) \quad$ down $\quad$ to $\quad \mathrm{SU}(3)^{2} \otimes \mathrm{SU}(5)^{3}$ $\otimes \mathrm{U}(1)^{6}$. [Note that $\mathrm{SO}(8)$ is broken to $\mathrm{SU}(3) \otimes \mathrm{U}(1)^{2}$, and each $\mathrm{SO}(10)$ is broken to $\mathrm{SU}(5) \otimes \mathrm{U}(1)]$. However, there are additional gauge bosons that come from the shifted and inverse shifted sectors. These are in the irreducible representations (irreps) $(\mathbf{3}, \overline{\mathbf{3}})(2)$ and $(\overline{\mathbf{3}}, \mathbf{3})(-2)$ of $\mathrm{SU}(3)$ $\otimes \mathrm{SU}(3) \otimes \mathrm{U}(1)$, where the $\mathrm{U}(1)$ charge (which is normalized to $1 / \sqrt{6}$ ) is given in the parentheses. Thus, the gauge symmetry of the $N 6$ model is $\mathrm{SU}(6) \otimes \mathrm{SU}(5)^{3} \otimes \mathrm{U}(1)^{5}$. This enhancement of gauge symmetry is made possible by breaking the $\mathrm{SO}(10)$ subgroups, so that the resulting $F 1$ model can have enhanced an hidden sector.

The final $F 1$ model is the $\mathbf{Z}_{6}$ orbifold $\left(T_{3}, T_{2}\right)$ of the $N 6$ model. When only the $T_{3}$ twist acts on the $N 6$ model, the resulting model has the gauge symmetry $\mathrm{SU}(4)_{1} \otimes \mathrm{SU}(5)_{3} \otimes \mathrm{U}(1)^{3}$. [Note that $\mathrm{SU}(6)$ is broken down to $\mathrm{SU}(4) \otimes \mathrm{U}(1)^{2}$, and four of the U(1)'s in the $N 6$ models have been removed by the $T_{3}$ twist.] The number of chiral families of $\mathrm{SU}(5)_{3}$ in this model is 9 , as is the case for other level-three models constructed from a single $\mathbf{Z}_{3}$ twist [5]. Therefore, we add the $T_{2}$ twist to obtain a model with three chiral families. The $F 1$ model has gauge symmetry $\mathrm{SU}(3)_{1} \otimes \mathrm{SU}(5)_{3} \otimes \mathrm{U}(1)^{4}$. Its massless spectrum is given in the first column of Table V. They are grouped according to where they come from, namely, the untwisted sector $U$, the $\mathbf{Z}_{3}$ twisted (i.e., $T_{3}$ and $2 T_{3}$ ) sector $T 3$, the $\mathbf{Z}_{6}$ twisted (i.e., $T_{3}+T_{2}$ and $2 T_{3}+T_{2}$ ) sector $T 6$, and $\mathbf{Z}_{2}$ twisted (i.e., $T_{2}$ ) sector $T 2$. Note that the $\mathrm{SU}(3)$ subgroup in the $F 1$ model arises as a result of the breaking $\mathrm{SU}(4) \supset \mathrm{SU}(3) \otimes \mathrm{U}(1)$. The net number of chiral families of $\mathrm{SU}(5)_{3}$ is 3 .

In the alternative procedure, we combine the Wilson line $A_{6}$ with the twists $\left(T_{3}, T_{2}\right)$ to form the $\mathbf{Z}_{3} \otimes \mathbf{Z}_{2} \otimes \mathbf{Z}_{3}$ orbifold generated by $\left(T_{3}, T_{2}, A_{6}\right)$, acting on the $N 1(1,0)$ model constructed above. Here we find that there are additional possibilities. Indeed, since the orders of $T_{3}$ and $A_{6}$ are the same (both of them have order 3), their respective contributions in the one-loop partition function can have a nontrivial relative phase between them. Let this phase be $\phi\left(T_{3}, A_{6}\right)$. This phase must satisfy $3 \phi\left(T_{3}, A_{6}\right)=0(\bmod 1)$ [i.e., $\phi\left(T_{3}, A_{6}\right)$ can be $0,1 / 3,2 / 3]$, and the states that survive the $T_{3}$ projection in the $A_{6}$ shifted sector must have the $T_{3}$ phase $\phi\left(T_{3}, A_{6}\right)$. Similarly, the states that survive the $T_{3}$ projection in the inverse shifted sector $\left(A_{6}\right)^{-1}$ must have the $T_{3}$ phase $-\phi\left(T_{3}, A_{6}\right)$. The string consistency then requires that the states that survive the $A_{6}$ projection in the $T_{3}$ twisted sector must have the $A_{6}$ phase $-\phi\left(T_{3}, A_{6}\right)$. Similarly, the states that survive the $A_{6}$ projection in the inverse twisted sector $\left(T_{3}\right)^{-1}$ must have the $A_{6}$ phase $\phi\left(T_{3}, A_{6}\right)$. The gauge symmetry of the resulting model depends on the choice of $\phi\left(T_{3}, A_{6}\right)$, since this phase dictates what states in these sectors are projected out. The models with $\phi\left(T_{3}, A_{6}\right)=0$ and $\phi\left(T_{3}, A_{6}\right)=1 / 3$ turn out to be equivalent, yielding precisely the $F 1$ model. The third choice $\phi\left(T_{3}, A_{6}\right)$ $=2 / 3$ leads to a different model, which we will refer to as the $F 2$ model. The $F 2$ model has gauge symmetry $\mathrm{SU}(2)_{1} \otimes \mathrm{SU}(2)_{1} \otimes \mathrm{SU}(5)_{3} \otimes \mathrm{U}(1)^{4}$, with the $\mathrm{SU}(2)_{1} \otimes \mathrm{SU}(2)_{1}$ forming the hidden sector. Its massless spectrum is also given in Table $\mathrm{V}$. The $F 1$ and the $F 2$ models were first constructed in [4]. In contrast to the $\mathrm{SO}(10)$ model, both of these two SU(5) models have an anomalous $\mathrm{U}(1)$ gauge symmetry. 
Since $A_{6}$ is a pure shift (without twist), the invariant sublattices and the numbers of fixed points in the $T_{3}$ and $T_{2}$ twists of the $F 1$ and the $F 2$ models remain the same as that in the $T 1(1,0)$ model. This allows us to borrow the construction of the $T 1(1,0)$ model to help working out the spectra of the $F 1$ and the $F 2$ models. This simplification trick will be used extensively in the next section.

\section{CLASSIFICATION OF SU(5) AND SU(6) MODELS}

In this section, we construct three-family $S U(5)$ and SU(6) models in the framework of $\mathbf{Z}_{6}$ or $\mathbf{Z}_{6} \otimes \mathbf{Z}_{3}$ asymmetric orbifolds. To achieve this, we give a classification of $\mathbf{Z}_{3}$ Wilson lines that can be added to the $\mathrm{SO}(10)$ and $\mathrm{E}_{6}$ models classified in [5] to obtain the corresponding SU(5) and SU(6) models. Models labeled with $N, E, T, S$, and $F$ are Narain, $\mathrm{E}_{6}$, $\mathrm{SO}(10)$, SU(6), and $\mathrm{SU}(5)$ string models, respectively. The massless spectra of many of the models are given in the tables. The massless spectra of the remaining models can be easliy obtained using the procudures presented below. Let us first start with the $E 1, E 2, T 1(1,1)$, and $T 2(1,1)$ models of [5]. The E1 model. The Wilson lines

$$
\begin{aligned}
& U_{1}=\left(0,0,0|| e_{1} / 2,0,0\right)(\mathbf{s}|\mathbf{0}| \mathbf{0} \mid \bar{S}), \\
& U_{2}=\left(0,0,0|| e_{2} / 2,0,0\right)(\mathbf{0}|\mathbf{s}| \mathbf{0} \mid \bar{S}),
\end{aligned}
$$

acting on the $N(1,1)$ model generate the $N 1(1,1)$ model, with $\mathrm{SU}(3)^{2} \otimes\left(\mathrm{E}_{6}\right)^{3}$ gauge symmetry. Start from this $N 1(1,1)$ model and perform the following twists:

$$
\begin{gathered}
T_{3}=\left(\theta, \theta, \theta|| \theta, e_{1} / 3,0\right)(\mathcal{P} \mid 2 / 3), \\
T_{2}=\left(\sigma, p_{1}, p_{2}|| 0, e_{1} / 2, e_{1} / 2\right)\left(0^{15} \mid 0\right) .
\end{gathered}
$$

This $E 1$ model has $\mathrm{SU}(2)_{1} \otimes\left(\mathrm{E}_{6}\right)_{3} \otimes \mathrm{U}(1)^{3}$ gauge symmetry.

The $E 2$ model. This model, which is the $T$ dual of the $E 1$ model, is obtained if we perform the following twists:

$$
\begin{gathered}
T_{3}=\left(0, \theta, \theta \| \theta, e_{1} / 3,0\right)(\mathcal{P} \mid 2 / 3), \\
T_{2}=\left(\sigma, p_{1}, p_{2}|| 0, e_{1} / 2, e_{1} / 2\right)\left(0^{15} \mid 0\right),
\end{gathered}
$$

on the $N 1(1,1)$ model.

The $T 1(1,1)$ model. The $N 2(1,1)$ model is generated by the Wilson lines

$$
\begin{aligned}
& U_{1}=\left(0, e_{1} / 2, e_{1} / 2|| e_{1} / 2,0,0\right)(\mathbf{s}|\mathbf{0}| \mathbf{0} \mid \bar{S}), \\
& U_{2}=\left(0, e_{2} / 2, e_{2} / 2|| e_{2} / 2,0,0\right)(\mathbf{0}|\mathbf{s}| \mathbf{0} \mid \bar{S}),
\end{aligned}
$$

acting on the $N(1,1)$ model. This $N 2(1,1)$ model has $\mathrm{SU}(3)^{2} \otimes \mathrm{U}(1)^{2} \otimes \mathrm{SO}(10)^{3} \otimes \mathrm{SO}(2)$ gauge symmetry. Start from the $N 2(1,1)$ model and perform the same twists as in the $E 1$ model. The resulting $T 1(1,1)$ model has $\mathrm{SU}(2)_{1} \otimes \mathrm{SO}(10)_{3} \otimes \mathrm{U}(1)^{4}$ gauge symmetry.

The $T 2(1,1)$ model. Start from the N2(1,1) model and perform the same twists as in the $E 2$ model. This model has $\mathrm{SU}(2)_{1} \otimes \mathrm{SO}(10)_{3} \otimes \mathrm{U}(1)^{4}$ gauge symmetry.

Now, one of the following Wilson lines can be added to the above models:
TABLE I. The massless spectra of the $S 2(1,0)$ and $F 1(1,1)$ models with gauge groups $\mathrm{SU}(2)_{1} \otimes \mathrm{SU}(2)_{3} \otimes \mathrm{SU}(6)_{3} \otimes \mathrm{U}(1)^{3}$ and (3) $\mathrm{SU}(2)_{1} \otimes \mathrm{SU}(5)_{3} \otimes \mathrm{U}(1)^{5}$, respectively. The $\mathrm{U}(1)$ normalization radii are given at the bottom of the table. The gravity, dilaton, and gauge supermultiplets are not shown.

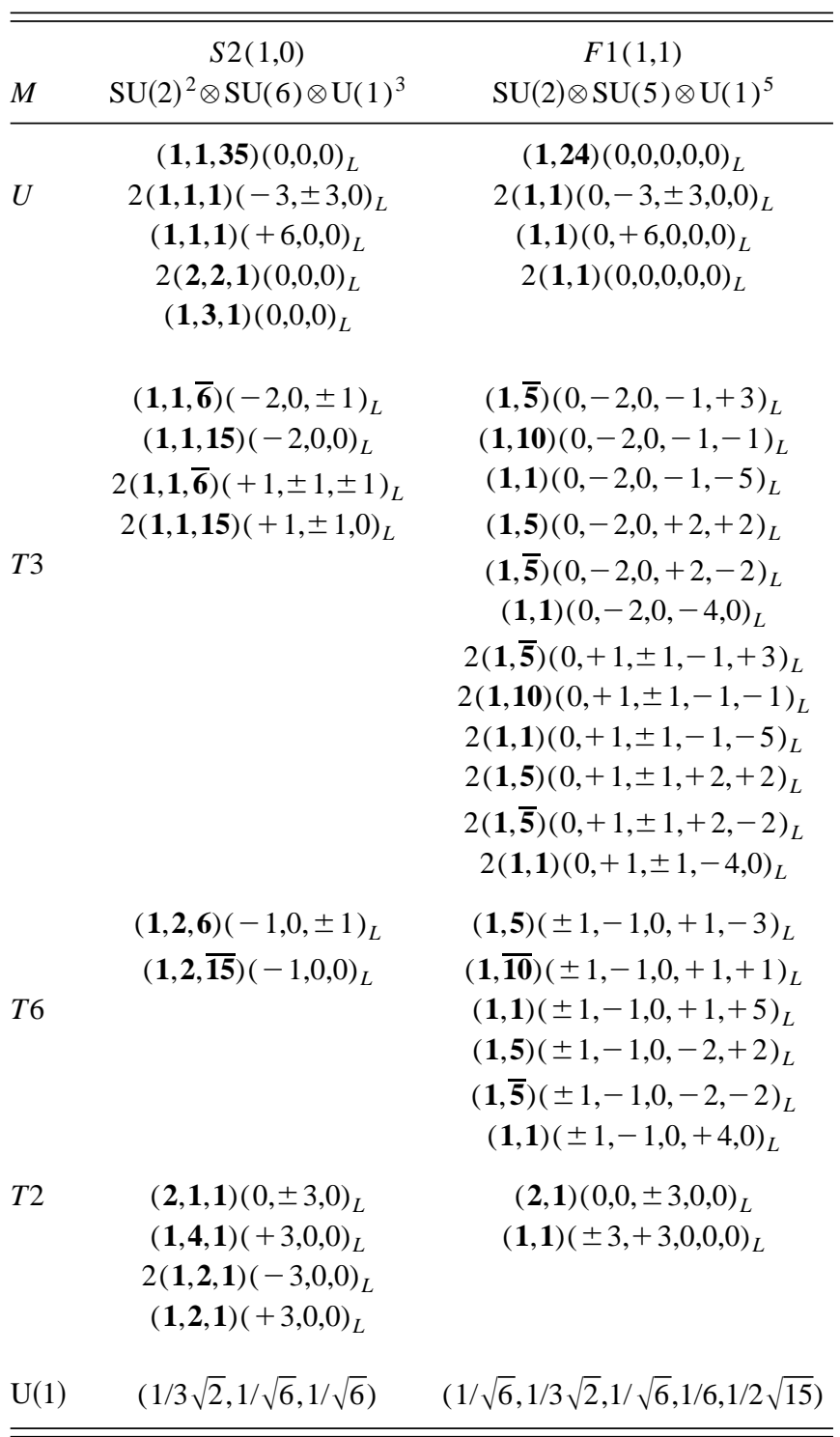

$$
A_{1}=\left(0,0,0 \| 0, \widetilde{e}^{2}, 0\right)\left(\left(\frac{1}{3} \frac{1}{3} \frac{1}{3} \frac{1}{3} \frac{2}{3}\right)^{3} \mid 0\right),
$$$$
A_{2}=\left(0,0,0|| 0,0, \widetilde{e^{2}}\right)\left(\left(\frac{1}{3} \frac{1}{3} \frac{1}{3} \frac{1}{3} \frac{2}{3}\right)^{3} \mid 0\right),
$$

$$
A_{3}=\left(0,0,0|| 0, \widetilde{e}^{2},-\widetilde{e}^{2}\right)\left(\left(\frac{1}{3} \frac{1}{3} \frac{1}{3} \frac{1}{3} \frac{2}{3}\right)^{3} \mid 0\right) \text {. }
$$

Adding one of these Wilson lines to one of the above models yields one of the following $\mathrm{SU}(6)$ and $\mathrm{SU}(5)$ models.

The $\mathrm{S} 2(1,0)$ model. Start from the $E 1$ model and add the $A_{2}$ Wilson line. This model has $\mathrm{SU}(2)_{1}$ $\otimes \mathrm{SU}(2)_{3} \otimes \mathrm{SU}(6)_{3} \otimes \mathrm{U}(1)^{3}$ gauge symmetry. The massless spectrum of the $S 2(1,0)$ model is given in Table I. Note that 
the spectrum of this model is very similar to that of the $T 2(1,0)$ model of [5]. In particular, the former can be obtained from the latter via replacing $\mathrm{SO}(10)_{3} \otimes \mathrm{U}(1)$ (the last $\mathrm{U}(1)$ normalized to $1 / 6$ in the second column of Table VII in [5]) by $\mathrm{SU}(6)_{3} \otimes \mathrm{U}(1)$ [the last $\mathrm{U}(1)$ normalized to $1 / \sqrt{6}$ in the first column of Table I]. Under this substitution, $\mathbf{4 5}(0)$ of $\mathrm{SO}(10)_{3} \otimes \mathrm{U}(1)$ is replaced by $35(0)$ of $\mathrm{SU}(6)_{3} \otimes \mathrm{U}(1)$, and $\mathbf{1 6}(-1)+\mathbf{1 0}(+2)+\mathbf{1}(-4)$ is replaced by $\mathbf{1 5}(0)+\overline{\mathbf{6}}(+1)$ $+\overline{\mathbf{6}}(-1)$. (The complex conjugates of these states are substituted similarly.) This replacement procedure will be used extensively below.

The $S 1(1,0)$ model. Start from the $E 1$ model and add the $A_{1} \quad$ Wilson line. This model has $\mathrm{SU}(2)_{1} \otimes \mathrm{SU}(2)_{3}$ $\otimes \mathrm{SU}(6)_{3} \otimes \mathrm{U}(1)^{3}$ gauge symmetry. The massless spectum of this model can be obtained from that of the $T 1(1,0)$ model by the replacement procedure described for the $S 2(1,0)$ model.

The $S 3(1,0)$ model. Start from the $E 2$ model and add the $A_{2} \quad$ Wilson line. This model has $\mathrm{SU}(2)_{1} \otimes \mathrm{SU}(2)_{3}$ $\otimes \mathrm{SU}(6)_{3} \otimes \mathrm{U}(1)^{3}$ gauge symmetry. The massless spectum of this model can be obtained from that of the $T 3(1,0)$ model by the replacement procedure described for the $S 2(1,0)$ model. Note that the $S 3(1,0)$ model is the same as the $S 1(1,0)$ model, i.e., $S 3(1,0)=S 1(1,0)$.

The $S 4(1,0)$ model. Start from the $E 2$ model and add the $A_{1} \quad$ Wilson line. This model has $\mathrm{SU}(2)_{1} \otimes \mathrm{SU}(2)_{3}$ $\otimes \mathrm{SU}(6)_{3} \otimes \mathrm{U}(1)^{3}$ gauge symmetry. The massless spectrum of this model can be obtained from that of the $T 4(1,0)$ model by the replacement procedure described for the $S 2(1,0)$ model. Note that $S 4(1,0)=S 2(1,0)$.

The $F 1(1,1)$ model. Start from the $T 1(1,1)$ model and add either the $A_{1}$ or $A_{2}$ Wilson line (both Wilson lines give the same model). This model has $\mathrm{SU}(2)_{1} \otimes \mathrm{SU}(5)_{3} \otimes \mathrm{U}(1)^{5}$ gauge symmetry. The massless spectrum of the $F 1(1,1)$ model is given in Table I. Note that the spectrum of this model is very similar to that of the $T 1(1,1)$ model of [5]. In particular, the former can be obtained from the latter via replacing $\mathrm{SO}(10)_{3}$ by $\mathrm{SU}(5)_{3} \otimes \mathrm{U}(1)$ [the last $\mathrm{U}(1)$ normalized to $1 / 2 \sqrt{15}$ in the second column of Table I]. Under this substitution, 45 of $\mathrm{SO}(10)_{3}$ is replaced by $\mathbf{2 4}(0)+\mathbf{1}(0)$ of $\mathrm{SU}(5)_{3} \otimes \mathrm{U}(1), \quad \mathbf{1 6}$ is replaced by $\overline{\mathbf{5}}(+3)+\mathbf{1 0}(-1)+$ $\mathbf{1}(-5), \mathbf{1 0}$ is replaced by $\mathbf{5}(+2)+\overline{\mathbf{5}}(-2)$, and the singlet $\mathbf{1}$ is replaced by $\mathbf{1}(0)$. This corresponds to the regular breaking $\mathrm{SO}(10) \supset \mathrm{SU}(5) \otimes \mathrm{U}(1)$.

The $F 2(1,1)$ model. Start from the $T 2(1,1)$ model and add either the $A_{1}$ or $A_{2}$ Wilson line (both Wilson lines give the same model). This model has $\mathrm{SU}(2)_{1} \otimes \mathrm{SU}(5)_{3} \otimes \mathrm{U}(1)^{5}$ gauge symmetry. The massless spectrum of the $F 2(1,1)$ model can be obtained from that of the $T 2(1,1)$ model by the breaking procedure described for the $F 1(1,1)$ model. Note that $F 2(1,1)=F 1(1,1)$.

The $S 1$ model. Start from the $T 1(1,1)$ model and add the $A_{3}$ Wilson line. Choose the relative phase between the $T_{3}$ and $A_{3}$ sectors to be $\phi\left(T_{3}, A_{3}\right)=0$ or $1 / 3$ (both choices give the same model). This model has $\mathrm{SU}(3)_{1} \otimes \mathrm{SU}(6)_{3} \otimes \mathrm{U}(1)^{3}$ gauge symmetry. The massless spectrum of the $S 1$ model is given in Table II.

The $S 2$ model. Start from the $T 1(1,1)$ model and add the $A_{3}$ Wilson line. Choose the relative phase between the $T_{3}$
TABLE II. The massless spectra of the two SU(6) models $S 1$ and $S 2$ with gauge symmetries $\mathrm{SU}(3)_{1} \otimes \mathrm{SU}(6)_{3} \otimes \mathrm{U}(1)^{3}$ and $\mathrm{SU}(2)_{1} \otimes \mathrm{SU}(2)_{1} \otimes \mathrm{SU}(6)_{3} \otimes \mathrm{U}(1)^{3}$, respectively. Note that double signs [as in $\left.(\mathbf{1}, \mathbf{2}, \mathbf{1})( \pm 1, \pm 3,-3)_{L}\right]$ are correlated. The $\mathrm{U}(1)$ normalization radii are given at the bottom of the table. The graviton, dilaton, and gauge supermultiplets are not shown.

\begin{tabular}{|c|c|c|}
\hline$M$ & $\begin{array}{c}S 1 \\
S U(3) \otimes S U(6) \otimes U(1)^{3}\end{array}$ & $\begin{array}{c}S 2 \\
\mathrm{SU}(2)^{2} \otimes \mathrm{SU}(6) \otimes \mathrm{U}(1)^{3}\end{array}$ \\
\hline$U$ & $\begin{array}{c}(\mathbf{1}, \mathbf{3 5})(0,0,0)_{L} \\
(\mathbf{1}, \mathbf{1})(0,0,0)_{L} \\
(\mathbf{1}, \mathbf{1})(+6,0,0)_{L} \\
2(\mathbf{1}, \mathbf{1})(-3, \pm 3, \pm 3)_{L} \\
2(\overline{\mathbf{3}}, \mathbf{1})(+3,-3,+1)_{L} \\
(\mathbf{3}, \mathbf{1})(0,0,-4)_{L}\end{array}$ & $\begin{array}{c}(\mathbf{1}, \mathbf{1}, \mathbf{3 5})(0,0,0)_{L} \\
(\mathbf{1}, \mathbf{1}, \mathbf{1})(0,0,0)_{L} \\
(\mathbf{1}, \mathbf{1}, \mathbf{1})(0,0,-6)_{L} \\
2(\mathbf{1}, \mathbf{2}, \mathbf{1})( \pm 1, \mp 3,+3)_{L} \\
(\mathbf{2}, \mathbf{1}, \mathbf{1})( \pm 2,0,+3)_{L}\end{array}$ \\
\hline$T 3$ & $\begin{array}{c}6(\mathbf{1}, \mathbf{1 5})(+1,-1,-1)_{L} \\
6(\mathbf{1}, \overline{\mathbf{6}})(-2,+1,-1)_{L} \\
3(\mathbf{1}, \overline{\mathbf{6}})(+1,0,+2)_{L}\end{array}$ & $\begin{array}{c}3(\mathbf{1}, \mathbf{1}, \mathbf{1 5})(0,0,+2)_{L} \\
6(\mathbf{1}, \mathbf{1}, \overline{\mathbf{6}})( \pm 1, \mp 1,-1)_{L}\end{array}$ \\
\hline$T 6$ & $\begin{array}{c}3(\mathbf{1}, \mathbf{6})(+2,+1,+1)_{L} \\
3(\mathbf{1}, \overline{\mathbf{1 5}})(-1,-1,+1)_{L}\end{array}$ & $3(\mathbf{1}, \mathbf{1}, \mathbf{6})( \pm 1, \pm 1,+1)_{L}$ \\
\hline$T 2$ & $\begin{array}{l}(\mathbf{3}, \mathbf{1})( \pm 3,-3,-1)_{L} \\
(\overline{\mathbf{3}}, \mathbf{1})(-3,+3,+1)_{L} \\
(\mathbf{1}, \mathbf{1})(+3, \pm 3, \mp 3)_{L}\end{array}$ & $\begin{array}{c}(\mathbf{2}, \mathbf{2}, \mathbf{1})( \pm 1, \mp 3,0)_{L} \\
(\mathbf{1}, \mathbf{2}, \mathbf{1})( \pm 1, \pm 3,-3)_{L}\end{array}$ \\
\hline $\mathrm{U}(1)$ & $\left(\frac{1}{3 \sqrt{2}}, \frac{1}{2 \sqrt{3}}, \frac{1}{2 \sqrt{3}}\right)$ & $\left(\frac{1}{2}, \frac{1}{2 \sqrt{3}}, \frac{1}{3 \sqrt{2}}\right)$ \\
\hline
\end{tabular}

and $A_{3}$ twisted sectors to be $\phi\left(T_{3}, A_{3}\right)=2 / 3$. This model has $\mathrm{SU}(2)_{1} \otimes \mathrm{SU}(2)_{1} \otimes \mathrm{SU}(6)_{3} \otimes \mathrm{U}(1)^{3}$ gauge symmetry. The massless spectrum of the $S 2$ model is given in Table II.

The $S 3$ model. Start from the $T 2(1,1)$ model and add the $A_{3}$ Wilson line. Choose the relative phase between the $T_{3}$ and $A_{3}$ twisted sectors to be $\phi\left(T_{3}, A_{3}\right)=0$ or $1 / 3$ (both choices give the same model). This model has $\mathrm{SU}(3)_{1} \otimes \mathrm{SU}(6)_{3} \otimes \mathrm{U}(1)^{3}$ gauge symmetry. The massless spectrum of the $S 3$ model is given in Table III. Note that $S 3=S 1$.

The $S 4$ model. Start from the $T 2(1,1)$ model and add the $A_{3}$ Wilson line. Choose the relative phase between the $T_{3}$ and $A_{3}$ twisted sectors to be $\phi\left(T_{3}, A_{3}\right)=2 / 3$. This model has $\mathrm{SU}(2)_{1} \otimes \mathrm{SU}(2)_{1} \otimes \mathrm{SU}(6)_{3} \otimes \mathrm{U}(1)^{3}$ gauge symmetry. The massless spectrum of the $S 4$ model is given in Table III. Note that $S 4=S 2$.

Here let us make a few comments for clarification. Note that adding the $A_{3}$ Wilson line to the $E 1$ and $E 2$ models does not result in three-family level-three grand unified string models. This can be seen from the fact that if we add the $A_{3}$ Wilson line to the $N 1(1,1)$ model of [5], the resulting Narain model will have gauge symmetry $\mathrm{SU}(6) \otimes \mathrm{SU}(18)$. The corresponding $\mathbf{Z}_{6}$ orbifold of the latter does not lead to a level-three model.

The following point is important for constructing other $\mathrm{SU}(6)$ models (see below). The gauge group of the $N(1,1)$ model is $\mathrm{E}_{6} \otimes \mathrm{SO}(32)$. This lattice itself can be conveniently 
TABLE III. The massless spectra of the two SU(5) models $S 3$ and $S 4$ with gauge symmetries $\mathrm{SU}(3)_{1} \otimes \mathrm{SU}(6)_{3} \otimes \mathrm{U}(1)^{3}$ and $\mathrm{SU}(2)_{1} \otimes \mathrm{SU}(2)_{1} \otimes \mathrm{SU}(6)_{3} \otimes \mathrm{U}(1)^{3}$, respectively. Note that double signs [as in $\left.(\mathbf{1}, \mathbf{2}, \mathbf{1})( \pm 1, \pm 3,-3)_{L}\right]$ are correlated. The $\mathrm{U}(1)$ normalization radii are given at the bottom of the table. The graviton, dilaton, and gauge supermultiplets are not shown.

\begin{tabular}{|c|c|c|}
\hline$M$ & $\begin{array}{c}S 3 \\
\mathrm{SU}(3) \otimes \mathrm{SU}(6) \otimes \mathrm{U}(1)^{3}\end{array}$ & $\begin{array}{c}S 4 \\
\mathrm{SU}(2)^{2} \otimes \mathrm{SU}(6) \otimes \mathrm{U}(1)^{3}\end{array}$ \\
\hline$U$ & $\begin{array}{c}(\mathbf{1}, \mathbf{3 5})(0,0,0)_{L} \\
(\mathbf{1}, \mathbf{1})(0,0,0)_{L} \\
(\mathbf{1}, \mathbf{1})(+6,0,0)_{L} \\
(\overline{\mathbf{3}}, \mathbf{1})(-3,+3,+1)_{L} \\
(\mathbf{1}, \mathbf{1})(+3, \pm 3, \mp 3)_{L} \\
(\mathbf{3 , 1})( \pm 3,-3,-1)_{L} \\
(\mathbf{3}, \mathbf{1})(0,0,-4)_{L}\end{array}$ & $\begin{array}{c}(\mathbf{1}, \mathbf{1}, \mathbf{3 5})(0,0,0)_{L} \\
(\mathbf{1}, \mathbf{1}, \mathbf{1})(0,0,0)_{L} \\
(\mathbf{1}, \mathbf{1}, \mathbf{1})(0,0,-6)_{L} \\
(\mathbf{1}, \mathbf{2}, \mathbf{1})( \pm 1, \pm 3,-3)_{L} \\
(\mathbf{2}, \mathbf{1}, \mathbf{1})( \pm 2,0,+3)_{L} \\
(\mathbf{2}, \mathbf{2}, \mathbf{1})( \pm 1, \mp 3,0)_{L}\end{array}$ \\
\hline$T 3$ & $\begin{array}{c}3(\mathbf{1}, \overline{\mathbf{1 5}})(-1,-1,+1)_{L} \\
3(\mathbf{1}, \mathbf{6})(+2,+1,+1)_{L} \\
3(\mathbf{1}, \overline{\mathbf{6}})(+1,0,+2)_{L}\end{array}$ & $\begin{array}{c}3(\mathbf{1}, \mathbf{1}, \mathbf{1 5})(0,0,+2)_{L} \\
3(\mathbf{1}, \mathbf{1}, \mathbf{6})( \pm 1, \pm 1,+1)_{L}\end{array}$ \\
\hline$T 6$ & $\begin{array}{l}6(\mathbf{1}, \overline{\mathbf{6}})(-2,+1,-1)_{L} \\
6(\mathbf{1}, \mathbf{1 5})(+1,-1,-1)_{L}\end{array}$ & $6(\mathbf{1}, \mathbf{1}, \overline{\mathbf{6}})( \pm 1, \mp 1,-1)_{L}$ \\
\hline$T 2$ & $\begin{array}{l}2(\mathbf{1}, \mathbf{1})(-3, \pm 3, \pm 3)_{L} \\
2(\overline{\mathbf{3}}, \mathbf{1})(+3,-3,+1)_{L}\end{array}$ & $2(\mathbf{1}, \mathbf{2}, \mathbf{1})( \pm 1, \mp 3,+3)_{L}$ \\
\hline $\mathrm{U}(1)$ & $\left(\frac{1}{3 \sqrt{2}}, \frac{1}{2 \sqrt{3}}, \frac{1}{2 \sqrt{3}}\right)$ & $\left(\frac{1}{2}, \frac{1}{2 \sqrt{3}}, \frac{1}{3 \sqrt{2}}\right)$ \\
\hline
\end{tabular}

viewed as being constructed from the $N(0,0)$ lattice by turning on the following Wilson line:

$$
U_{3}=\left(0,0,0||, \widetilde{e}^{2},-\widetilde{e^{2}}, \widetilde{e}^{2}\right)\left(0^{15} \mid 0\right) .
$$

Then the corresponding $N 1(1,1)$ and $N 2(1,1)$ models can be viewed as being constructed from the $N(0,0)$ lattice by turning on the $U_{3}$ Wilson line along with the appropriate $U_{1}$ and $U_{2}$ Wilson lines. Now, the Wilson lines $A_{1}, A_{2}, A_{3}$, and $U_{3}$ are all order 3 shifts, so it is possible to have nonzero relative phases between them. The relevant relative phases are $\phi\left(A_{i}, U_{3}\right)$, where $i=1,2,3$. In the above description of models $S 1-4(1,0), F 1(1,1), F 2(1,1)$, and $S 1-4$, we have set these phases to be zero. If we let $\phi\left(A_{i}, U_{3}\right)$ take nonzero values, i.e., $1 / 3$ or $2 / 3$, we obtain the same set of models. For example, in the construction of the $S 1$ model, if we had taken $\phi\left(A_{3}, U_{3}\right) \neq 0$ instead of $\phi\left(A_{3}, U_{3}\right)=0$, we would end up with the $F 1(1,1)$ model. So no new model is generated with this approach. However, there is another Wilson line,

$$
A_{3}^{\prime}=\left(0,0,0|| 0, \widetilde{e^{2}}, \widetilde{e^{2}}\right)\left(\left(\frac{1}{3} \frac{1}{3} \frac{1}{3} \frac{1}{3} \frac{2}{3}\right)^{3} \mid 0\right),
$$

for which new models will emerge. More precisely, if we add this Wilson line to the $E 1$ and $E 2$ models, and set the phase $\phi\left(A_{3}^{\prime}, U_{3}\right)$ to zero, we recover the $S 1-4$ models discussed previously. If we add this Wilson line to the
TABLE IV. The massless spectra of the $S 1(1,1)$ and $F 11(1,0)$ models with gauge groups $\mathrm{SU}(2)_{1} \otimes \mathrm{SU}(6)_{3} \otimes \mathrm{U}(1)^{4}$ and $\mathrm{SU}(2)_{1} \otimes \mathrm{SU}(2)_{3} \otimes \mathrm{SU}(5)_{3} \otimes \mathrm{U}(1)^{4}$, respectively. The U(1) normalization radii are given at the bottom of the table. The gravity, dila-

\begin{tabular}{|c|c|c|}
\hline$M$ & $\begin{array}{c}S 1(1,1) \\
\mathrm{SU}(2) \otimes \mathrm{SU}(6) \otimes \mathrm{U}(1)^{4}\end{array}$ & $\begin{array}{c}F 11(1,0) \\
\mathrm{SU}(2)^{2} \otimes \mathrm{SU}(5) \otimes \mathrm{U}(1)^{4}\end{array}$ \\
\hline & $(\mathbf{1}, \mathbf{3 5})(0,0,0,0)_{L}$ & $(\mathbf{1}, \mathbf{1}, \mathbf{2 4})(0,0,0,0)_{L}$ \\
\hline \multirow[t]{4}{*}{$U$} & $\begin{array}{c}2(\mathbf{1}, \mathbf{1}, \mathbf{1})(0,-3, \pm 3,0)_{L} \\
\quad(\mathbf{1}, \mathbf{1}, \mathbf{1})(0,+6,0,0)_{L} \\
\quad(\mathbf{1}, \mathbf{1}, \mathbf{1})(0,0,0,0)_{L}\end{array}$ & $\begin{array}{c}2(\mathbf{1}, \mathbf{1}, \mathbf{1})(0,-3, \pm 3,0)_{L} \\
\quad(\mathbf{1}, \mathbf{1}, \mathbf{1})(0,+6,0,0)_{L} \\
\quad(\mathbf{1}, \mathbf{1}, \mathbf{1})(0,0,0,0)_{L} \\
\quad(\mathbf{1}, \mathbf{3}, \mathbf{1})(0,0,0,0)_{L}\end{array}$ \\
\hline & $(\mathbf{1}, \mathbf{1 5})(0,-2,0,0)_{L}$ & $(\mathbf{1}, \mathbf{2}, \overline{\mathbf{5}})(0,-2,0,+1)_{L}$ \\
\hline & $(\mathbf{1}, \overline{\mathbf{6}})(0,-2,0,+1)_{L}$ & $(\mathbf{1}, \mathbf{2}, \mathbf{1})(0,-2,0,-5)_{L}$ \\
\hline & $(\mathbf{1}, \overline{\mathbf{6}})(0,-2,0,-1)_{L}$ & $\begin{array}{c}(\mathbf{1}, \mathbf{1}, \mathbf{1 0})(0,-2,0,-2)_{L} \\
(\mathbf{1}, \mathbf{1}, \mathbf{5})(0,-2,0,+4)_{L}\end{array}$ \\
\hline \multirow[t]{6}{*}{$T 3$} & $2(\mathbf{1}, \mathbf{5})(0,+1, \pm 1,0)_{L}$ & $2(\mathbf{1}, \mathbf{2}, \overline{\mathbf{5}})(0,+1, \pm 1,+1)_{L}$ \\
\hline & $2(\mathbf{1}, \overline{\mathbf{6}})(0,+1, \pm 1,+1)_{L}$ & $2(\mathbf{1}, \mathbf{2}, \mathbf{1})(0,+1, \pm 1,-5)_{L}$ \\
\hline & $2(\mathbf{1}, \overline{\mathbf{6}})(0,+1, \pm 1,-1)_{L}$ & $\begin{array}{c}2(\mathbf{1}, \mathbf{1}, \mathbf{1 0})(0,+1, \pm 1,-2)_{L} \\
2(\mathbf{1}, \mathbf{1}, \mathbf{5})(0,+1, \pm 1,+4)_{L}\end{array}$ \\
\hline & $(\mathbf{1}, \overline{\mathbf{1 5}})( \pm 1,-1,0,0)_{L}$ & $(\mathbf{1}, \mathbf{2}, \mathbf{5})( \pm 1,-1,0,-1)_{L}$ \\
\hline & $(\mathbf{1}, \mathbf{6})( \pm 1,-1,0,+1)_{L}$ & $(\mathbf{1}, \mathbf{2}, \mathbf{1})( \pm 1,-1,0,+5)_{L}$ \\
\hline & $(\mathbf{1}, \mathbf{6})( \pm 1,-1,0,-1)_{L}$ & $\begin{array}{c}(\mathbf{1}, \mathbf{1}, \overline{\mathbf{1 0}})( \pm 1,-1,0,+2)_{L} \\
(\mathbf{1}, \mathbf{1}, \overline{\mathbf{5}})( \pm 1,-1,0,-4)_{L}\end{array}$ \\
\hline$T 2$ & $\begin{array}{c}(\mathbf{2}, \mathbf{1}, \mathbf{1})(0,0, \pm 3,0)_{L} \\
(\mathbf{1}, \mathbf{1}, \mathbf{1})( \pm 3,+3,0,0)_{L}\end{array}$ & $\begin{array}{c}(\mathbf{2}, \mathbf{1}, \mathbf{1})(0,0, \pm 3,0)_{L} \\
(\mathbf{1}, \mathbf{1}, \mathbf{1})( \pm 3,+3,0,0)_{L}\end{array}$ \\
\hline $\mathrm{U}(1)$ & $(1 / \sqrt{6}, 1 / 3 \sqrt{2}, 1 / \sqrt{6}, 1 / \sqrt{6})$ & $(1 / \sqrt{6}, 1 / 3 \sqrt{2}, 1 / \sqrt{6}, 1 / 3 \sqrt{10})$ \\
\hline
\end{tabular}
ton, and gauge supermultiplets are not shown.

$T 1(1,1)$ and $T 2(1,1)$ models, and set the phase $\phi\left(A_{3}^{\prime}, U_{3}\right)$ to zero, we recover the $F 1-4$ models discussed below. If, in the latter case, we take the phase $\phi\left(A_{3}^{\prime}, U_{3}\right) \neq 0$, we recover the models $F 1(1,1)$ and $F 2(1,1)$. Now, if we add this Wilson line to the $E 1$ and $E 2$ models, and take the phase $\phi\left(A_{3}^{\prime}, U_{3}\right) \neq 0$, we find two new models, which we will refer to as $S 5(1,1)$ and $S 6(1,1)$, respectively. Now, let us describe these models.

The $S 1(1,1)$ model. Start from the $E 1$ model and add the $A_{3}^{\prime}$ Wilson line. Choose the relative phase $\phi\left(A_{3}^{\prime}, U_{3}\right)=1 / 3$ or $2 / 3$ (both choices give the same model). This model has $\mathrm{SU}(2)_{1} \otimes \mathrm{SU}(6)_{3} \otimes \mathrm{U}(1)^{4}$ gauge symmetry. The massless spectrum of the $S 1(1,1)$ model is given in Table IV. Note that the spectrum of this model is very similar to that of the $E 1$ model. In particular, the former can be obtained from the latter via replacing $\left(\mathrm{E}_{6}\right)_{3}$ by $\mathrm{SU}(6)_{3} \otimes \mathrm{U}(1)$ [the last $\mathrm{U}(1)$ normalized to $1 / \sqrt{6}$ in the first column of Table IV). Under this substitution, 78 of $\left(E_{6}\right)_{3}$ is replaced by $35(0)+\mathbf{1}(0)$ of $\mathrm{SU}(6)_{3} \otimes \mathrm{U}(1), \quad$ and 27 is replaced by $\mathbf{1 5}+\overline{\mathbf{6}}(+1)+$ $\overline{\mathbf{6}}(-1)$. (The complex conjugates of these states are substituted similarly.) This corresponds to the regular breaking $\mathrm{E}_{6} \supset \mathrm{SU}(6) \otimes \mathrm{U}(1)$.

The $S 2(1,1)$ model. Start from the $E 1$ model and add the $A_{3}^{\prime}$ Wilson line. Choose the relative phase $\phi\left(A_{3}^{\prime}, U_{3}\right)=1 / 3$ or 
2/3 (both choices give the same model). This model has $\mathrm{SU}(2)_{1} \otimes \mathrm{SU}(6)_{3} \otimes \mathrm{U}(1)^{4}$ gauge symmetry. The massless spectrum of the $S 2(1,1)$ model can be obtained from that of the $E 2$ model by the breaking procedure described for the $S 1(1,1)$ model. Note that the $S 2(1,1)$ model is the same as the $S 1(1,1)$ model. Here we note that the $S 1(1,1)$ and $S 2(1,1)$ models can be obtained from the $T 1(1,1)$ and $T 2(1,1)$ models of [5] by the replacement procedure described for the $S 2(1,0)$ model. (Similarly, one may construct other $\mathrm{SU}(6)$ models whose massless spectra would follow from those of the corresponding $\mathrm{SO}(10)$ models given in [5] by the the replacement procedure described for the $S 2(1,0)$ model.)

Next, consider the models $T 1(0,0)$ to $T 11(0,0)$, and $T 1(1,0)$ to $T 10(1,0)$ of $[5]$. The following Wilson line can be added to all of these models:

$$
A_{4}=\left(0,0,0|| \widetilde{e}^{2}, 0,0\right)\left(\left(\frac{1}{3} \frac{1}{3} \frac{1}{3} \frac{1}{3} \frac{2}{3}\right)^{3} \mid 0\right) .
$$

We will refer to the resulting models as $F 1(0,0)$ to $F 11(0,0)$ and $F 1(1,0)$ to $F 10(1,0)$, respectively. These models have $\mathrm{SU}(5)_{3}$ grand unified gauge group. The massless spectra of these models can be obtained from those of the models $T 1(0,0)$ to $T 11(0,0)$, and $T 1(1,0)$ to $T 10(1,0)$ by the breaking procedure described for the $F 1(1,1)$ model. Note that some of these SU(5) models are the same just as some of the corresponding $\mathrm{SO}(10)$ models are the same. For example, $\quad F 3(1,0)=F 1(1,0)$, which follows from $T 3(1,0)=T 1(1,0)$. Here we note that, in the case of the $T 5(1,0)=T 6(1,0)$ model, the left-moving $\mathrm{SO}(8)$ momenta in the $A_{4}$ Wilson line are given in the $\mathrm{SO}(8)$ $\supset \mathrm{SU}(3) \otimes \mathrm{U}(1)^{2}$ basis. In [5] we gave the twists for these models with the left-moving $\mathrm{SO}(8)$ momenta being written in the $\mathrm{SO}(8) \supset \mathrm{SU}(2)^{4}$ basis. It is not difficult to translate between these two bases. In particular, the $A_{4}$ Wilson line in the latter basis reads

$$
A_{4}=\left(0,0,0|| \widetilde{e}^{2} \mid 0,0,0,0\right)\left(\left(\frac{1}{3} \frac{1}{3} \frac{1}{3} \frac{1}{3} \frac{2}{3}\right)^{3} \mid 0\right) .
$$

In [5] it was pointed out that the models $T 1(1,0), T 2(1,0)$, $T 3(1,0)$, and $T 4(1,0)$ can be obtained via asymmetric orbifolds. Here we will give these asymmetric orbifolds once again as they will be important for constructing new SU(5) models.

The $T 1(1,0)$ model. Start from the $N 1(1,0)$ model of [5] and perform the following twists:

$$
\begin{gathered}
T_{3}=\left(\theta, \theta, \theta \| 0, e_{1} / 3, \theta\right)(\mathcal{P} \mid 2 / 3), \\
T_{2}=\left(0, \sigma, \sigma \| e_{1} / 2, e_{1} / 2,0\right)\left(0^{15} \mid 0\right) .
\end{gathered}
$$

This model has $\mathrm{SU}(2)_{1} \otimes \mathrm{SU}(2)_{3} \otimes \mathrm{SO}(10)_{3} \otimes \mathrm{U}(1)^{3}$ gauge symmetry. The massless spectrum of the $T 1(1,0)$ model is given in Table VII of [5]. Note that this construction is different from the one presented in Sec. III. The equivalence of these two constructions are thoroughly explained in [3].

The $T 2(1,0)$ model. Start from the N1(1,0) model of [5] and perform the following twists:

$$
T_{3}=\left(\theta, \theta, \theta|| e_{1} / 3,0, \theta\right)(\mathcal{P} \mid 2 / 3),
$$

$$
T_{2}=\left(0, \sigma, \sigma|| e_{1} / 2, e_{1} / 2,0\right)\left(0^{15} \mid 0\right) .
$$

This model has $\mathrm{SU}(2)_{1} \otimes \mathrm{SU}(2)_{3} \otimes \mathrm{SO}(10)_{3} \otimes \mathrm{U}(1)^{3}$ gauge symmetry. The massless spectrum of the $T 2(1,0)$ model is given in Table VII of [5].

The $T 3(1,0)$ model. Start from the $N 1(1,0)$ model of [5] and perform the following twists:

$$
\begin{gathered}
T_{3}=\left(\theta, 0, \theta \| e_{1} / 3,0, \theta\right)(\mathcal{P} \mid 2 / 3), \\
T_{2}=\left(0, \sigma, \sigma \| e_{1} / 2, e_{1} / 2,0\right)\left(0^{15} \mid 0\right) .
\end{gathered}
$$

This model has $\mathrm{SU}(2)_{1} \otimes \mathrm{SU}(2)_{3} \otimes \mathrm{SO}(10)_{3} \otimes \mathrm{U}(1)^{3}$ gauge symmetry. The massless spectrum of the $T 3(1,0)$ model is given in Table VIII of [5].

The $T 4(1,0)$ model. Start from the $N 1(1,0)$ model of [5] and perform the following twists:

$$
\begin{gathered}
T_{3}=\left(\theta, 0, \theta|| 0, e_{1} / 3, \theta\right)(\mathcal{P} \mid 2 / 3), \\
T_{2}=\left(0, \sigma, \sigma|| e_{1} / 2, e_{1} / 2,0\right)\left(0^{15} \mid 0\right) .
\end{gathered}
$$

This model has $\mathrm{SU}(2)_{1} \otimes \mathrm{SU}(2)_{3} \otimes \mathrm{SO}(10)_{3} \otimes \mathrm{U}(1)^{3}$ gauge symmetry. The massless spectrum of the $T 4(1,0)$ model is given in Table VIII of [5].

The $T 7-10(1,0)$ models. If, instead of the $N 1(1,0)$ model, we start from the $N 2(1,0)$ model and perform the above twists for the $T 1-4(1,0)$ models, we obtain the $T 7-10(1,0)$ models of [5]. The models $T 1-4(1,0)$ and $T 7-10(1,0)$ now admit two more Wilson lines:

$$
A_{5}=\left(0,0,0 \| 0, \widetilde{e}^{2}, 0\right)\left(\left(\frac{1}{1} \frac{1}{3} \frac{1}{3} \frac{1}{3} \frac{2}{3}\right)^{3} \mid 0\right),
$$

$$
A_{6}=\left(0,0,0|| \widetilde{e}^{2},-\widetilde{e}^{2}, 0\right)\left(\left(\frac{1}{3} \frac{1}{3} \frac{1}{3} \frac{1}{3} \frac{2}{3}\right)^{3} \mid 0\right)
$$

Adding these Wilson lines, we obtain the following SU(5) models.

The $F 11(1,0)$ model. Start from either the $T 1(1,0)$ or $T 2(1,0)$ model (both choices give the same model) and add the $A_{5}$ Wilson line. This model has $\mathrm{SU}(2)_{1} \otimes \mathrm{SU}(2)_{3}$ $\otimes \mathrm{SU}(5)_{3} \otimes \mathrm{U}(1)^{4}$ gauge symmetry. The massless spectrum of the F11(1,0) model is given in Table IV. Note that the spectrum of this model is very similar to that of the $E 1$ model. In particular, the former can be obtained from the latter via replacing $\left(E_{6}\right)_{3}$ by $\mathrm{SU}(5)_{3} \otimes \mathrm{SU}(2)_{3} \otimes \mathrm{U}(1)$ [the last $\mathrm{U}(1)$ normalized to $1 / \sqrt{6}$ in the second column of Table IV]. Under this substitution, 78 of $\left(E_{6}\right)_{3}$ is replaced by $(\mathbf{2 4 , 1})(0)+(\mathbf{1}, \mathbf{3})(0)+(\mathbf{1}, \mathbf{1})(0)$ of $\mathrm{SU}(5)_{3}$ $\otimes \mathrm{SU}(2)_{3} \otimes \mathrm{U}(1)$, and 27 is replaced by $(\mathbf{1 0}, \mathbf{1})(-2)$ $+(\mathbf{5}, \mathbf{1})(+4)+(\overline{\mathbf{5}}, \mathbf{2})(+1)+(\mathbf{1}, \mathbf{2})(-5)$. (The complex conjugates of these states are substituted similarly.) This corresponds to the regular breaking $\mathrm{E}_{6} \supset \mathrm{SU}(5)$ $\otimes \mathrm{SU}(2) \otimes \mathrm{U}(1)$.

The $F 12(1,0)$ model. Start from either the T3(1,0) or $T 4(1,0)$ model (both choices give the same model) and add 
the $A_{5}$ Wilson line. This model has $\mathrm{SU}(2)_{1} \otimes \mathrm{SU}(2)_{3}$ $\otimes \mathrm{SU}(5)_{3} \otimes \mathrm{U}(1)^{4}$ gauge symmetry. The massless spectrum of the $F 12(1,0)$ model can be obtained from that of the $E 2$ model by the breaking procedure described for the $F 11(1,0)$ model. Note that $F 12(1,0)=F 11(1,0)$.

The $F 13(1,0)$ model. Start from either the $T 7(1,0)$ or $T 8(1,0)$ model (both choices give the same model) and add the $A_{5}$ Wilson line. This model has $\mathrm{SU}(2)_{1} \otimes \mathrm{SU}(2)_{3}$ $\otimes \mathrm{SU}(5)_{3} \otimes \mathrm{U}(1)^{4}$ gauge symmetry. The massless spectrum of the $F 13(1,0)$ model is the same as that of the $F 11(1,0)$ model with additional states coming from the $T_{2}$ sector. These states read $4(\mathbf{2}, \mathbf{1}, \mathbf{1})(0, \pm 3,0,0)_{L}$.

The $F 14(1,0)$ model. Start from either the $T 9(1,0)$ or $T 10(1,0)$ model (both choices give the same model) and add the $A_{5}$ Wilson line. This model has $\mathrm{SU}(2)_{1} \otimes \mathrm{SU}(2)_{3}$ $\otimes \mathrm{SU}(5)_{3} \otimes \mathrm{U}(1)^{4}$ gauge symmetry. The massless spectrum of the $F 14(1,0)$ model is the same as that of the $F 12(1,0)$ model with additional states coming from the $T_{2}$ sector. These states read $4(\mathbf{2}, \mathbf{1}, \mathbf{1})(0, \pm 3,0,0)_{L}$. Note that $F 14(1,0)=F 13(1,0)$.

The $F 1$ model. Start from either the $T 1(1,0)$ or $T 2(1,0)$ model (both choices give the same model) and add the $A_{6}$ Wilson line. Choose the relative phase between the $T_{3}$ and $A_{6}$ twisted sectors to be $\phi\left(T_{3}, A_{6}\right)=0$ or $1 / 3$ (both choices give the same model). This model has $\mathrm{SU}(3)_{1} \otimes \mathrm{SU}(5)_{3}$ $\otimes \mathrm{U}(1)^{4}$ gauge symmetry. The massless spectrum of the $F 1$ model is given in Table $\mathrm{V}$.

The $F 2$ model. Start from either the $T 1(1,0)$ or $T 2(1,0)$ model (both choices give the same model) and add the $A_{6}$ Wilson line. Choose the relative phase between the $T_{3}$ and $A_{6}$ twisted sectors to be $\phi\left(T_{3}, A_{6}\right)=2 / 3$. This model has $\mathrm{SU}(2)_{1} \otimes \mathrm{SU}(2)_{1} \otimes \mathrm{SU}(5)_{3} \otimes \mathrm{U}(1)^{4}$ gauge symmetry. The massless spectrum of the $F 2$ model is given in Table $\mathrm{V}$.

The $F 3$ model. Start from either the $T 3(1,0)$ or $T 4(1,0)$ model (both choices give the same model) and add the $A_{6}$ Wilson line. Choose the relative phase between the $T_{3}$ and $A_{6}$ twisted sectors to be $\phi\left(T_{3}, A_{6}\right)=0$ or $1 / 3$ (both choices give the same model). This model has $\mathrm{SU}(3)_{1} \otimes \mathrm{SU}(5)_{3}$ $\otimes \mathrm{U}(1)^{4}$ gauge symmetry. The massless spectrum of the $F 3$ model is given in Table VI. Note that $F 3=F 1(1,0)$.

The $F 4$ model. Start from either the $T 3(1,0)$ or $T 4(1,0)$ model (both choices give the same model) and add the $A_{6}$ Wilson line. Choose the relative phase between the $T_{3}$ and $A_{6}$ twisted sectors to be $\phi\left(T_{3}, A_{6}\right)=2 / 3$. This model has $\mathrm{SU}(2)_{1} \otimes \mathrm{SU}(2)_{1} \otimes \mathrm{SU}(5)_{3} \otimes \mathrm{U}(1)^{4}$ gauge symmetry. The massless spectrum of the $F 4$ model is given in Table VI. Note that $F 4=F 2(1,0)$.

The $F 5$ model. Start from either the $T 7(1,0)$ or $T 8(1,0)$ model (both choices give the same model) and add the $A_{6}$ Wilson line. Choose the relative phase between the $T_{3}$ and $A_{6}$ twisted sectors to be $\phi\left(T_{3}, A_{6}\right)=0$ or $1 / 3$ (both choices give the same model). This model has $\mathrm{SU}(3)_{1} \otimes \mathrm{SU}(5)_{3}$ $\otimes \mathrm{U}(1)^{4}$ gauge symmetry. The massless spectrum of the $F 5$ model is the same as that of the $F 1$ model with additional states coming from the $T_{2}$ sector. These additional states are given in Table VII.

The $F 6$ model. Start from either the $T 7(1,0)$ or $T 8(1,0)$ model (both choices give the same model) and add the $A_{6}$ Wilson line. Choose the relative phase between the $T_{3}$ and $A_{6}$ twisted sectors to be $\phi\left(T_{3}, A_{6}\right)=2 / 3$. This model has
TABLE V. The massless spectra of the two SU(5) models $F 1$ and $F 2$ with gauge symmetries $\mathrm{SU}(3)_{1} \otimes \mathrm{SU}(5)_{3} \otimes \mathrm{U}(1)^{4}$ and $\mathrm{SU}(2)_{1} \otimes \mathrm{SU}(2)_{1} \otimes \mathrm{SU}(5)_{3} \otimes \mathrm{U}(1)^{4}$, respectively. Note that double signs [as in $\left.(\mathbf{1}, \mathbf{2}, \mathbf{1})( \pm 1, \pm 3,-3,0)_{L}\right]$ are correlated. The $U(1)$ normalization radii are given at the bottom of the table. The graviton, dilaton, and gauge supermultiplets are not shown.

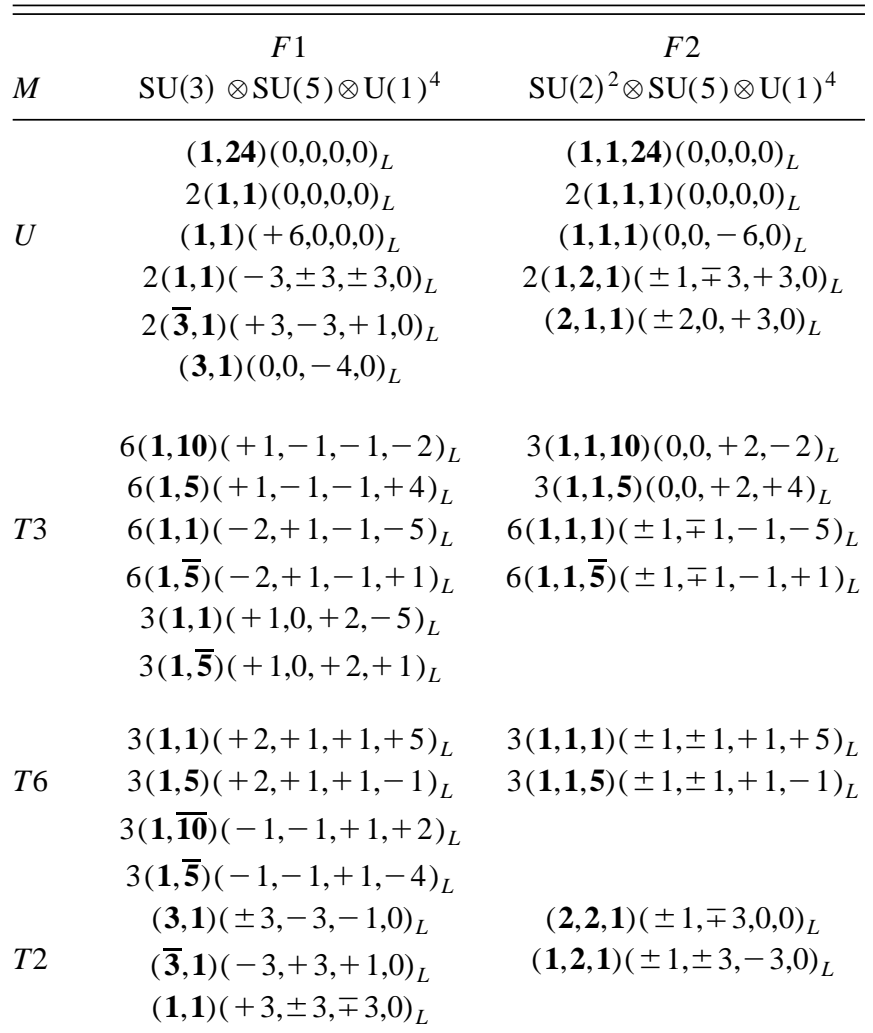

U(1) $\left(\frac{1}{3 \sqrt{2}}, \frac{1}{2 \sqrt{3}}, \frac{1}{2 \sqrt{3}}, \frac{1}{3 \sqrt{10}}\right) \quad\left(\frac{1}{2}, \frac{1}{2 \sqrt{3}}, \frac{1}{3 \sqrt{2}}, \frac{1}{3 \sqrt{10}}\right)$

$\mathrm{SU}(2)_{1} \otimes \mathrm{SU}(2)_{1} \otimes \mathrm{SU}(5)_{3} \otimes \mathrm{U}(1)^{4}$ gauge symmetry. The massless spectrum of the $F 6$ model is the same as that of the $F 2$ model with additional states coming from the $T_{2}$ sector. These additional states are given in Table VII.

The $F 7$ model. Start from either the $T 9(1,0)$ or $T 10(1,0)$ model (both choices give the same model) and add the $A_{6}$ Wilson line. Choose the relative phase between the $T_{3}$ and $A_{6}$ twisted sectors to be $\phi\left(T_{3}, A_{6}\right)=0$ or $1 / 3$ (both choices give the same model). This model has $\mathrm{SU}(3)_{1} \otimes \mathrm{SU}(5)_{3} \otimes \mathrm{U}(1)^{4}$ gauge symmetry. The massless spectrum of the $F 7$ model is the same as that of the $F 3$ model with additional states coming from the $T_{2}$ sector. These additional states are given in Table VII. Note that $F 7=F 5$.

The $F 8$ model. Start from either the $T 9(1,0)$ or $T 10(1,0)$ model (both choices give the same model) and add the $A_{6}$ Wilson line. Choose the relative phase between the $T_{3}$ and $A_{6}$ twisted sectors to be $\phi\left(T_{3}, A_{6}\right)=2 / 3$. This model has $\mathrm{SU}(2)_{1} \otimes \mathrm{SU}(2)_{1} \otimes \mathrm{SU}(5)_{3} \otimes \mathrm{U}(1)^{4}$ gauge symmetry. The massless spectrum of the $F 8$ model is the same as that of the $F 4$ model with additional states coming from the $T_{2}$ sector. These additional states are given in Table VII. Note 
TABLE VI. The massless spectra of the two SU(5) models $F 3$ and $F 4$ with gauge symmetries $\mathrm{SU}(3)_{1} \otimes \mathrm{SU}(5)_{3} \otimes \mathrm{U}(1)^{4}$ and $\mathrm{SU}(2)_{1} \otimes \mathrm{SU}(2)_{1} \otimes \mathrm{SU}(5)_{3} \otimes \mathrm{U}(1)^{4}$, respectively. Note that double signs [as in $\left.(\mathbf{1}, \mathbf{2}, \mathbf{1})( \pm 1, \pm 3,-3,0)_{L}\right]$ are correlated. The $\mathrm{U}(1)$ normalization radii are given at the bottom of the table. The graviton, dilaton, and gauge supermultiplets are not shown.

\begin{tabular}{|c|c|c|}
\hline$M$ & $\begin{array}{c}F 3 \\
\mathrm{SU}(3) \otimes \mathrm{SU}(5) \otimes \mathrm{U}(1)^{4}\end{array}$ & $\begin{array}{c}F 4 \\
\mathrm{SU}(2)^{2} \otimes \mathrm{SU}(5) \otimes \mathrm{U}(1)^{4}\end{array}$ \\
\hline$U$ & $\begin{array}{c}(\mathbf{1}, \mathbf{2 4})(0,0,0,0)_{L} \\
2(\mathbf{1}, \mathbf{1})(0,0,0,0)_{L} \\
(\mathbf{1}, \mathbf{1})(+6,0,0,0)_{L} \\
(\mathbf{3}, \mathbf{1})( \pm 3,-3,-1,0)_{L} \\
(\overline{\mathbf{3}}, \mathbf{1})(-3,+3,+1,0)_{L} \\
(\mathbf{1}, \mathbf{1})(+3, \pm 3, \mp 3,0)_{L} \\
(\mathbf{3}, \mathbf{1})(0,0,-4,0)_{L}\end{array}$ & $\begin{array}{c}(\mathbf{1}, \mathbf{1}, \mathbf{2 4})(0,0,0,0)_{L} \\
2(\mathbf{1}, \mathbf{1}, \mathbf{1})(0,0,0,0)_{L} \\
(\mathbf{1}, \mathbf{1}, \mathbf{1})(0,0,-6,0)_{L} \\
(\mathbf{2}, \mathbf{2}, \mathbf{1})( \pm 1, \mp 3,0,0)_{L} \\
(\mathbf{1}, \mathbf{2}, \mathbf{1})( \pm 1, \pm 3,-3,0)_{L} \\
(\mathbf{2}, \mathbf{1}, \mathbf{1})( \pm 2,0,+3,0)_{L}\end{array}$ \\
\hline$T 3$ & $\begin{array}{c}3(\mathbf{1}, \mathbf{1})(+2,+1,+1,+5)_{L} \\
3(\mathbf{1}, \mathbf{5})(+2,+1,+1,-1)_{L} \\
3(\mathbf{1}, \overline{\mathbf{1 0}})(-1,-1,+1,+2)_{L} \\
3(\mathbf{1}, \overline{\mathbf{5}})(-1,-1,+1,-4)_{L} \\
3(\mathbf{1}, \mathbf{1})(+1,0,+2,-5)_{L} \\
3(\mathbf{1}, \overline{\mathbf{5}})(+1,0,+2,+1)_{L}\end{array}$ & $\begin{array}{c}3(\mathbf{1}, \mathbf{1}, \mathbf{1})( \pm 1, \pm 1,+1,+5)_{L} \\
3(\mathbf{1}, \mathbf{1}, \mathbf{5})( \pm 1, \pm 1,+1,-1)_{L} \\
3(\mathbf{1}, \mathbf{1}, \mathbf{1 0})(0,0,+2,-2)_{L} \\
3(\mathbf{1}, \mathbf{1}, \mathbf{5})(0,0,+2,+4)_{L}\end{array}$ \\
\hline$T 6$ & $\begin{array}{l}6(\mathbf{1}, \mathbf{1 0})(+1,-1,-1,-2)_{L} \\
6(\mathbf{1}, \mathbf{5})(+1,-1,-1,+4)_{L} \\
6(\mathbf{1}, \mathbf{1})(-2,+1,-1,-5)_{L} \\
6(\mathbf{1}, \overline{\mathbf{5}})(-2,+1,-1,+1)_{L}\end{array}$ & $\begin{array}{c}6(\mathbf{1}, \mathbf{1}, \mathbf{1})( \pm 1, \mp 1,-1,-5)_{L} \\
6(\mathbf{1}, \mathbf{1}, \overline{\mathbf{5}})( \pm 1, \mp 1,-1,+1)_{L}\end{array}$ \\
\hline$T 2$ & $\begin{array}{l}2(\mathbf{1}, \mathbf{1})(-3, \pm 3, \pm 3,0)_{L} \\
2(\overline{\mathbf{3}}, \mathbf{1})(+3,-3,+1,0)_{L}\end{array}$ & $2(\mathbf{1}, \mathbf{2}, \mathbf{1})( \pm 1, \mp 3,+3,0)_{L}$ \\
\hline $\mathrm{U}(1)$ & $\left.\frac{1}{3 \sqrt{2}}, \frac{1}{2 \sqrt{3}}, \frac{1}{2 \sqrt{3}}, \frac{1}{3 \sqrt{10}}\right)$ & $\left(\frac{1}{2}, \frac{1}{2 \sqrt{3}}, \frac{1}{3 \sqrt{2}}, \frac{1}{3 \sqrt{10}}\right)$ \\
\hline
\end{tabular}

that $F 8=F 6$.

Next, consider the $T 5(1,0)$ and $T 6(1,0)$ models of [5]. These models admit the $A_{4}$ Wilson line. The resulting models are $F 5(1,0)$ and $F 6(1,0)$, as we disscussed previously. There are, however, additional Wilson lines that can be added to these two models. The $T 6(1,0)$ model admits the following four Wilson lines [here the left-moving $\mathrm{SO}(8)$ momenta are given in the $\mathrm{SO}(8) \supset \mathrm{SU}(2)^{4}$ basis]:

$$
\begin{gathered}
B_{1}=\left(0,0,0|| \widetilde{e}^{2} \mid, 0, \sqrt{2} / 3, \sqrt{2} / 3,-\sqrt{2} / 3\right)\left(\left(\frac{1}{3} \frac{1}{3} \frac{1}{3} \frac{1}{3} \frac{2}{3}\right)^{3} \mid 0\right), \\
B_{2}=(0,0,0|| 0 \mid, 0, \sqrt{2} / 3, \sqrt{2} / 3,-\sqrt{2} / 3)\left(\left(\frac{1}{3} \frac{1}{3} \frac{1}{3} \frac{1}{3} \frac{2}{3}\right)^{3} \mid 0\right), \\
B_{3}=\left(0,0,0|| e_{1} / 3 \mid-\sqrt{2} / 3, \sqrt{2} / 3,0,0\right)\left(\left(\frac{1}{3} \frac{1}{3} \frac{1}{3} \frac{1}{3} \frac{2}{3}\right)^{3} \mid 0\right), \\
B_{4}=\left(0,0,0|| e_{1} / 3 \mid 0,0, \sqrt{2} / 3,-\sqrt{2} / 3\right)\left(\left(\frac{1}{3} \frac{1}{3} \frac{1}{3} \frac{1}{3} \frac{2}{3}\right)^{3} \mid 0\right) .
\end{gathered}
$$

The $T 5(1,0)$ model admits the Wilson lines $B_{1}, B_{2}$, and $B_{3}$, but not $B_{4}$. A priori, there are three cases to consider for each Wilson line, namely, there are three choices of the relative phases $\phi\left(T_{3}, B_{i}\right)$. For the sake of brevity, we will not list all the possible models. However, let us consider one of these models for illustration.

The $F 9$ model. Start from the $T 6(1,0)$ model and add the $B_{1}$ Wilson line. Choose the relative phase between the $T_{3}$ and $B_{1}$ sectors to be $\phi\left(T_{3}, B_{1}\right)=2 / 3$. This model has $\mathrm{SU}(4)_{1} \otimes \mathrm{SU}(2)_{1} \otimes \mathrm{SU}(5)_{1} \otimes \mathrm{U}(1)^{4}$ gauge symmetry. The massless spectrum of this model is given in Table VIII. Note that $\mathrm{SU}(4)_{1}$ is the hidden sector gauge group in this model, whereas $\mathrm{SU}(2)_{1}$ is the horizontal symmetry gauge group. Also note that this model is completely anomaly free.

Here we note that some of the models obtained by adding the above $B_{i}$ Wilson lines to the $T 5(1,0)$ and $T 6(1,0)$ models do not have any hidden sector, although some of them do possess non-Abelian horizontal gauge symmetries [these are products of $\left.\mathrm{SU}(2)_{1}\right]$. Examples of such models are the following. Start from the T5(1,0) model and add either the $B_{1}$ or $B_{2}$ Wilson line. Then, regardless of the choice of $\phi\left(T_{3}, B_{i}\right)$, the resulting model does not have a hidden sector. Another point that we should stress is that some of the models have anomalous U(1)'s in their spectra. An example of this is the following. Start from the T6 $(1,0)$ model and add the $B_{1}$ Wilson line. Choose the relative phase between the $T_{3}$ and $B_{1}$ sectors to be $\phi\left(T_{3}, B_{1}\right)=0$ or $2 / 3$ (both choices give the same model). This model has $\mathrm{SU}(2)_{1} \otimes \mathrm{SU}(2)_{1}$ $\otimes \mathrm{SU}(5)_{1} \otimes \mathrm{U}(1)^{6}$ gauge symmetry. Here $\mathrm{SU}(2)_{1} \otimes \mathrm{SU}(2)_{1}$ is the hidden sector gauge group. This model has an anomalous U(1). The generic feature of all of these models is that they are $\mathrm{SU}(5)_{3}$ grand unified string models with three families of quarks and leptons, and they have only one adjoint (and no other higher dimensional representation) Higgs field,

TABLE VII. The states to be added to the massless spectra of the models $F 1$ and $F 3$ to obtain the massless spectra of the models $F 5$ and $F 7$ (first column), and to the $F 2$ and $F 4$ to obtain the massless spectra of the models $F 6$ and $F 8$ (second column), respectively. The corresponding gauge groups are given at the top of the table. The $\mathrm{U}(1)$ normalization radii are given at the bottom of the table. The additional states shown in the table appear in the $T 2$ sector.

\begin{tabular}{ccc}
\hline \hline & $F 5, F 7$ & $F 6, F 8$ \\
& $\mathrm{SU}(3)_{1} \otimes \mathrm{SU}(5)_{3} \otimes \mathrm{U}(1)^{4}$ & $\mathrm{SU}(2)_{1} \otimes \mathrm{SU}(2)_{1} \otimes \mathrm{SU}(5)_{3} \otimes \mathrm{U}(1)^{4}$ \\
\hline $2(\mathbf{3}, \mathbf{1})(0,0,+2,0)_{L}$ & $4(\mathbf{2}, \mathbf{1}, \mathbf{1})(0,0, \pm 3,0)_{L}$ \\
$\mathrm{U}(1)$ & $4(\overline{\mathbf{3}}, \mathbf{1})(0,0,-2,0)_{L}$ & $4(\mathbf{1}, \mathbf{1}, \mathbf{1})( \pm 2,0,0,0)_{L}$ \\
\hline \hline
\end{tabular}


TABLE VIII. The massless spectrum of the $F 9$ model with gauge group $\mathrm{SU}(4)_{1} \otimes \mathrm{SU}(2)_{1} \otimes \mathrm{SU}(5)_{3} \otimes \mathrm{U}(1)^{4}$. The $\mathrm{U}(1)$ normalization radii are given at the bottom of the table. The gravity, dilaton, and gauge supermultiplets are not shown.

\begin{tabular}{|c|c|}
\hline$M$ & $\begin{array}{c}F 9 \\
\mathrm{SU}(4) \otimes \mathrm{SU}(2) \otimes \mathrm{SU}(5) \otimes \mathrm{U}(1)^{4}\end{array}$ \\
\hline$U$ & $\begin{array}{c}(\mathbf{1}, \mathbf{1}, \mathbf{2 4})(0,0,0,0)_{L} \\
(\mathbf{1}, \mathbf{1}, \mathbf{1})(0,0,0,0)_{L} \\
(\mathbf{4}, \mathbf{2}, \mathbf{1})(0,0,0,+3)_{L} \\
(\overline{\mathbf{4}}, \mathbf{2}, \mathbf{1})(0,0,0,-3)_{L}\end{array}$ \\
\hline$T 3$ & $\begin{aligned}(\mathbf{1}, \mathbf{1}, \overline{\mathbf{1 0}})(+2,-2,+2,0)_{L} & (\mathbf{1}, \mathbf{1}, \overline{\mathbf{5}})(+2,-2,-4,0)_{L} \\
(\mathbf{1}, \mathbf{1}, \overline{\mathbf{1 0}})(+2,+2,+2,0)_{L} & (\mathbf{1}, \mathbf{1}, \overline{\mathbf{5}})(+2,+2,-4,0)_{L} \\
(\mathbf{1}, \mathbf{1}, \overline{\mathbf{1 0}})(-4,0,+2,0)_{L} & (\mathbf{1}, \mathbf{1}, \overline{\mathbf{5}})(-4,0,-4,0)_{L}\end{aligned}$ \\
\hline$T 6$ & $\begin{aligned}(\mathbf{1}, \mathbf{1}, \mathbf{1 0})(-2,0,-2, \pm 2)_{L} & (\mathbf{1}, \mathbf{1}, \mathbf{5})(-2,0,+4, \pm 2)_{L} \\
(\mathbf{1}, \mathbf{1}, \mathbf{1 0})(+1,-1,-2, \pm 2)_{L} & (\mathbf{1}, \mathbf{1}, \mathbf{5})(+1,-1,+4, \pm 2)_{L} \\
(\mathbf{1}, \mathbf{1}, \mathbf{1 0})(+1,+1,-2, \pm 2)_{L} & (\mathbf{1}, \mathbf{1}, \mathbf{5})(+1,+1,+4, \pm 2)_{L} \\
(\mathbf{1}, \mathbf{2}, \overline{\mathbf{5}})(-2,0,+1,0)_{L} & (\mathbf{1}, \mathbf{1}, \mathbf{1})(-2,0,-5,0)_{L} \\
(\mathbf{1}, \mathbf{2}, \overline{\mathbf{5}})(+1,-1,+1,0)_{L} & (\mathbf{1}, \mathbf{1}, \mathbf{1})(+1,-1,-5,0)_{L} \\
(\mathbf{1}, \mathbf{2}, \overline{\mathbf{5}})(+1,+1,+1,0)_{L} & (\mathbf{1}, \mathbf{1}, \mathbf{1})(+1,+1,-5,0)_{L}\end{aligned}$ \\
\hline$T 2$ & $\begin{array}{c}(\mathbf{4}, \mathbf{2}, \mathbf{1})(0,0,0,-3)_{L} \\
(\overline{\mathbf{4}}, \mathbf{2}, \mathbf{1})(0,0,0,+3)_{L} \\
(\mathbf{6}, \mathbf{1}, \mathbf{1})(+6,0,0,0)_{L} \\
(\mathbf{6}, \mathbf{1}, \mathbf{1})(-3,+3,0,0)_{L} \\
(\mathbf{6}, \mathbf{1}, \mathbf{1})(-3,-3,0,0)_{L}\end{array}$ \\
\hline $\mathrm{U}(1)$ & $(1 / 6,1 / 2 \sqrt{3}, 1 / 3 \sqrt{10}, 1 / 2 \sqrt{3})$ \\
\hline
\end{tabular}

and the adjoint Higgs field does not carry any gauge quantum numbers other than those of the grand unified gauge group. One adjoint Higgs field neutral under other gauge quantum numbers is a generic feature for all three-family models constructed in this paper and in [5].

Finally, let us summarize the new versus old results presented in this paper. The models $F 1(1,1)$ and $S 2(1,0)$ were first presented in [3]. There the possibility of having an enhanced hidden sector gauge group was also pointed out. The first examples of models with enhanced hidden sectors were constructed in [4]. In particular, [4] presented the models $F 1, F 2$, and briefly discussed the $S 1$ and $S 2$ models. The rest of the models classified in this paper are new. This concludes the construction of three-family SU(5) and SU(6) string models. Together with [5], the present paper completes the construction of three-family grand unification in string theory.

\section{MODULI SPACE OF SU(5) AND SU(6) MODELS}

In [5], we pointed out that all but one of the $\mathrm{SO}(10)$ models constructed there are connected by flat moduli. Such connections also exist for most of the SU(5) and SU(6) models constructed in this paper as well. Let us collect them into three sets.

(i) Starting from Fig. 1 of [5], the same picture will hold if we replace all the $S O(10)$ models there by the correspond-
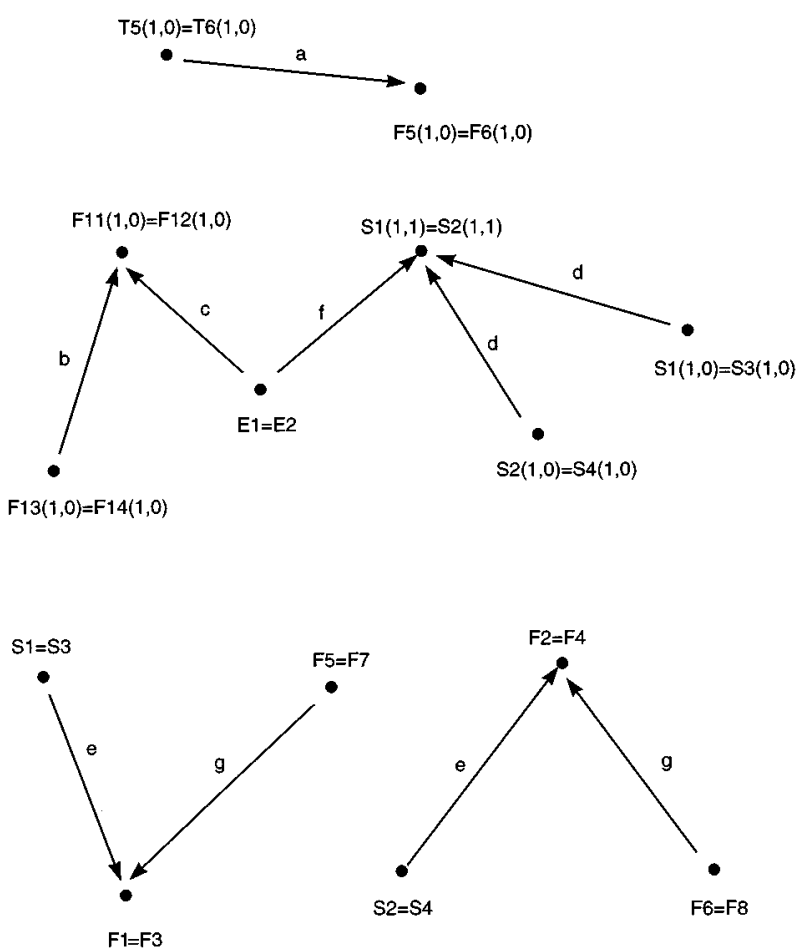

FIG. 1. The arrows correspond to flat directions. Here $a, b, c$, $d, e, f$, and $g$ stand for the fields whose VEV's are the corresponding flat directions. See Section V for details.

ing $\mathrm{SU}(5)$ models, and the $E 1=E 2$ model by the $T 1(1,1)=T 2(1,1)$ model, respectively,

(ii) Starting from Fig. 1 of [5], the same picture will hold if we replace all the $\mathrm{SO}(10)$ models there by the corresponding $\mathrm{SU}(6)$ models, while keeping the $E 1=E 2$ model untouched. Since the $E 1=E 2$ model is connected to the $T 1(1,1)=T 2(1,1)$ model, these two sets are connected as well.

(iii) The rest of the connections are summarized in Fig. 1 in this paper. There, the symbols $a, b, c, d, e, f, g$ stand for the fields whose vevs are the corresponding flat directions. Thus, $a$ corresponds to the adjoint breaking of $\mathrm{SO}(10)$ down to $\mathrm{SU}(5) \otimes \mathrm{U}(1) ; b$ and $g$ stand for VEV's of singlets, i.e., neutral under all the gauge symmetries; $c$ corresponds to the adjoint breaking of $\mathrm{E}_{6}$ down to $\mathrm{SU}(5) \otimes \mathrm{SU}(2) \otimes \mathrm{U}(1) ; d$ stands for the triplet Higgs [i.e., $(\mathbf{1 , 3}, \mathbf{1})(0,0,0)_{L}$ ] of the $\mathrm{SU}(2)_{3}$ subgroup in models $S 1(1,0)=S 3(1,0)$ and $S 2(1,0)=S 4(1,0) ; e$ corresponds to the adjoint breaking of $\mathrm{SU}(6)$ down to $\mathrm{SU}(5) \otimes \mathrm{U}(1)$; finally, $f$ stands for the adjoint breaking of $E_{6}$ down to $\mathrm{SU}(6) \otimes \mathrm{U}(1)$.

This completes our discussion of the moduli space of three-family SU(5) and SU(6) string models constructed in this paper, and their connections to the $\mathrm{SO}(10)$ and the $\mathrm{E}_{6}$ models.

\section{REMARKS}

Together, [5] and this paper give the classification of all $\mathbf{Z}_{6}$ asymmetric orbifolds that can yield three-family supersymmetric grand unified string models that have a nonAbelian hidden sector. The massless spectra of many of these models are presented in the tables in these two papers. Note 
that the number of models increases as the rank of the GUT gauge symmetry decreases: there is a unique $\mathrm{E}_{6}$ model, but there are dozens of SU(5) models. This is expected, since a smaller GUT symmetry permits more room for the hidden and the intermediate sectors, so the number of choices for the latter increases.

It remains an open question if this is a complete classification of all three-family GUT models within the framework of conformal field theory and asymmetric orbifolds. One possibility that is outside of our framework is the the nondiagonal embedding of the $\mathrm{SU}(5)_{3}$ current algebra. Although we have argued that it is unlikely that other higher-level realizations of the current algebras can yield interesting three-family GUT models, it will be important to explore them as well. Presumably, three-family GUT string models can also be obtained in nonperturbative string theory. This direction requires a better understanding of string theory.

Phenomenologically, are any of the three-family GUT string models constructed so far viable? There are many is- sues that one has to address before one can come to some sort of an answer to this question. The first step is clearly to determine the couplings of any specific model. This is certainly within the reach of our present understanding. Knowing the superpotential, or at least some of its leading terms, will definitely help. This knowledge may also shed light on how these GUT models differ from the more conventional standard model strings.

\section{ACKNOWLEDGMENTS}

We would like to thank Michael Bershadsky, Pran Nath, Gary Shiu, Tom Taylor, and Yan Vtorov-Karevsky for discussions. The research of S.-H.H.T. was partially supported by National Science Foundation. The work of Z.K. was supported in part by Grant No. NSF PHY-96-02074, and the U.S. DOE. Z.K. would also like to thank Albert $\mathrm{Yu}$ and Ribena $\mathrm{Yu}$ for financial support.
[1] See, e.g., D. C. Lewellen, Nucl. Phys. B337, 61 (1990); J. A. Schwartz, Phys. Rev. D 42, 1777 (1990); S. Chaudhuri, S.-W. Chung, G. Hockney, and J. D. Lykken, Nucl. Phys. B456, 89 (1995); G. B. Cleaver, ibid. B456, 219 (1995); G. Aldazabal, A. Font, L. E. Ibáñez, and A. M. Uranga, ibid. B452, 3 (1995); J. Erler, ibid. B475, 597 (1996); Z. Kakushadze, G. Shiu, and S.-H. H. Tye, Phys. Rev. D 54, 7545 (1996).

[2] Z. Kakushadze and S.-H. H. Tye, Phys. Rev. Lett. 77, 2612 (1996).

[3] Z. Kakushadze and S.-H. H. Tye, Phys. Rev. D 54, 7520 (1996).

[4] Z. Kakushadze and S.-H. H. Tye, Phys. Lett. B 392, 335 (1996)

[5] Z. Kakushadze and S.-H. H. Tye, preceding paper, Phys. Rev. D 55, 7878 (1997).

[6] L. Dixon, J. Harvey, C. Vafa, and E. Witten, Nucl. Phys. B261, 620 (1985); B274, 285 (1986); K. S. Narain, M. H. Sarmadi, and C. Vafa, ibid. B288 551 (1987); L. E. Ibáñez, H. P. Nilles, and F. Quevedo, Phys. Lett. B 187, 25 (1987).

[7] On superstring phenomenology, see, e.g., J.-P. Derendinger, L. E. Ibáñez, and H. P. Nilles, Phys. Lett. 155B, 65 (1985); M. Dine, R. Rohm, N. Seiberg, and E. Witten, ibid. 156B, 55 (1985); T. Taylor, G. Veneziano, and S. Yankielowicz, Nucl. Phys. B218, 493 (1983); T. Taylor, Phys. Lett. 164B, 43
(1985); A. Font, L. E. Ibáñez, D. Lüst, and F. Quevedo, ibid. 245, 401 (1990); S. Ferrara, N. Magnoli, T. Taylor, and G. Veneziano, ibid. 245, 409 (1990); H. P. Nilles and M. Olechowski, ibid. 248, 268 (1990); P. Binétruy and M. K. Gaillard, ibid. 253, 119 (1991); L. Dixon, V. Kaplunovsky, and J. Louis, Nucl. Phys. B329, 27 (1990); B355, 649 (1991); V. Kaplunovsky, ibid. B307, 145 (1988); B382, 436 (1992); V. Kaplunovsky and J. Louis, ibid. B444, 191 (1995).

[8] K. R. Dienes and J. March-Russell, Nucl. Phys. B479, 113 (1996); K. R. Dienes, Report No. IASSNS-HEP-96/64 hep-ph/9606467, 1996 (unpublished).

[9] M. Green and J. H. Schwarz, Phys. Lett. 149B, 117 (1984); E. Witten, ibid. 149B, 351 (1984); M. Dine, N. Seiberg, and E. Witten, Nucl. Phys. B289, 585 (1987); J. Attick, L. Dixon, and A. Sen, ibid. B292, 109 (1987); M. Dine, I. Ichinose, and N. Seiberg, B293, 253 (1987).

[10] N. V. Krasnikov, Phys. Lett. B 193, 37 (1987); L. Dixon, V. Kaplunovsky, J. Louis, and M. Peskin, SLAC Report No. SLAC-PUB-5229, 1990 (unpublished); J. A. Casas, Z. Lalak, C. Muñoz, and G. G. Ross, Nucl. Phys. B347, 243 (1990); T. Taylor, Phys. Lett. B 252, 59 (1990).

[11] K. S. Narain, Phys. Lett. 169B, 41 (1986); K. S. Narain, M. H. Sarmadi, and E. Witten, Nucl. Phys. B279, 369 (1987). 